\title{
A VIRTUAL ELEMENT DISCRETIZATION FOR THE TIME DEPENDENT NAVIER-STOKES EQUATIONS IN STREAM-FUNCTION FORMULATION
}

\author{
Dibyendu Adak ${ }^{1,2, *} \oplus$, David Mora ${ }^{1,3}$, Sundararajan Natarajan $^{2}$ \\ and Alberth Silgado ${ }^{1}$
}

\begin{abstract}
In this work, a new Virtual Element Method (VEM) of arbitrary order $k \geq 2$ for the time dependent Navier-Stokes equations in stream-function form is proposed and analyzed. Using suitable projection operators, the bilinear and trilinear terms are discretized by only using the proposed degrees of freedom associated with the virtual space. Under certain assumptions on the computational domain, error estimations are derived and shown that the method is optimally convergent in both space and time variables. Finally, to justify the theoretical analysis, four benchmark examples are examined numerically.
\end{abstract}

Mathematics Subject Classification. 65N30, 65N12, 76D07, 65N15.

Received April 4, 2021. Accepted September 15, 2021.

\section{INTRODUCTION}

In this work, we study a Virtual Element Method (VEM) for a fourth order nonlinear problem arising in the numerical discretization of the Navier-Stokes problem. The VEM, introduced in [6], is a generalization of the Finite Element Method (FEM) which is characterized by the capability of dealing with very general polygonal/polyhedral meshes, and it also permits to construct in a straightforward way highly regular discrete spaces. Indeed, by avoiding the explicit construction of the local basis functions, the VEM can easily handle general polygons/polyhedrons without complex integrations on the element (see [7] for details on the coding aspects of the method). The VEM has been applied successfully for problems in fluid mechanics; see for instance $[1,8,17,18,24,26,30,33,34,39,41]$, where Stokes, Brinkman, Stokes-Darcy and Navier-Stokes equations have been recently developed.

The Navier-Stokes system is a paradigm of fluid flow problems. Usually, the variables $\boldsymbol{u}$ and $p$ denote the velocity and the pressure field, respectively. It is proved that if the body force $\mathbf{f}$ and the initial data $\boldsymbol{u}_{0}$ are smooth enough and the boundary of domain $\Omega$ is locally Lipschitz continuous, then the two dimensional non stationary Navier-Stokes problem has weak solution. In [38], Temam showed that $\boldsymbol{u} \in L^{\infty}\left(0, T ;\left[H^{2}(\Omega)\right]^{2}\right)$ with the assumption that $\mathbf{f}$ and initial data $\boldsymbol{u}_{0}$ are suitably smooth. Since the model problem consists of nonlinear term, it is not straightforward to find analytical solution. Therefore, numerical approximation is

Keywords and phrases. Virtual element method, Navier-Stokes equations, time dependent problem, stream-function.

1 GIMNAP, Departamento de Matemática, Universidad del Bío-Bío, Concepción, Chile.

2 Department of Mechanical Engineering, Indian Institute of Technology Madras, Chennai 600036, India.

$3 \mathrm{CI}^{2} \mathrm{MA}$, Universidad de Concepción, Concepción, Chile.

* Corresponding author: dadak@ubiobio.cl, dibyendu.jumath@gmail.com

(c) The authors. Published by EDP Sciences, SMAI 2021

This is an Open Access article distributed under the terms of the Creative Commons Attribution License (https://creativecommons.org/licenses/by/4.0), which permits unrestricted use, distribution, and reproduction in any medium, provided the original work is properly cited. 
the only available option in the majority of the cases found in practice, which involves very difficult initial and boundary conditions. A large amount of articles are contributed to improve the effectiveness of numerical schemes, the computer resolution of Navier-Stokes equations still challenges the applied mathematician and scientist. In this article, we have attempted to develop a VEM scheme for a time dependent stream-function formulation of the Navier-Stokes equations.

In the proposed formulation, the stream-function is the principal unknown of the system [27,28]. Salient features in formulations of this kind include that: there is only one scalar variable, the incompressible condition is satisfied automatically, the stream-function is one of the most useful tools in flow visualization. On the other hand, we note that the velocity and pressure are not present in the formulation. However, the velocity can be easily obtained by a simple postprocess from stream-function. In 1979 in [27] the standard weak formulation was presented for the first time in terms of the stream-function for the Navier-Stokes equations, in this direction in [20] the authors present conforming finite element method for the steady formulation, including an algorithm for pressure recovery. More recently, in [25] a $C^{1}$ finite element method based on the Argyris element has been proposed for the stationary quasi-geostrophic equations, which corresponds to an extension of a stream-function formulation for the Navier-Stokes problem.

It is well-known that conforming finite element methods to solve the fourth order problems require $C^{1}$ continuity. The construction of finite elements with such regularity is not straightforward (see [23]). However, this can be easily achieved by using the virtual element strategy. More precisely, we will follow the VEM approach presented in $[16,21]$ (see also $[10,12,35,36]$ ) to build global discrete spaces of $H^{2}(\Omega)$ of arbitrary order to solve the time dependent fourth order nonlinear problem.

There are some works for the approximation by VEM for fluid flow problems using the stream-function formulation. In [4] a $C^{1}$ conforming virtual element method has been presented to solve the Stokes problem on general polygonal meshes. More recently, a 2D Stokes complex structure for the VEM was analyzed and a discrete curl formulation of the Navier-Stokes problem has been obtained in [11]. The extension to the 3D case of the Stokes complex structure for the VEM has been presented in [13].

The goal of this paper is to propose a conforming $C^{1}$ virtual element method to solve continuous weak formulation (2.5) (will be defined in Sect. 2) and to prove that the method is optimally convergent in both space and time variables. More precisely, we will propose a new VEM discretization to solve the time dependent NavierStokes problem written in terms of the stream-function variable. We consider a primal variational formulation of the problem written in $H^{2}(\Omega)$. Then, we propose a direct $C^{1}$ global virtual element subspace of arbitrary order $k \geq 2$ to be used in the semi-discrete and fully-discrete formulations. We construct projection operators in order to write bilinear and trilinear forms that are fully computable. In particular, to discretize the trilinear form, we propose a form which does not need any stabilization. We prove that the fully-discrete problem is well-posed by using fixed point arguments and assuming that the data is in a certain sense small enough. Then, we obtain optimal rate of convergence in $H^{2}(\Omega)$ for the proposed discretizations by using an adequate projection operator and under standard assumptions on the computational domain. In addition, the velocity field is then obtained from the discrete stream-function by a postprocess. In a summary, the advantages of the present method are: the $C^{1}$ conforming virtual space can be built with a straightforward construction due to the flexibility of the VEM and it provides a very competitive alternative to solve the time dependent Navier-Stokes problem on polygonal meshes.

The rest of the paper is organized as follows: In Section 2, the model problem and the continuous weak formulation are defined. Using the stream-function formulation, we rewrite the continuous weak formulation that is nonlinear time dependent biharmonic problem. Further, the basic settings of the functional analysis and the assumptions required to develop the theory are also highlighted. In Section 3, we introduce the virtual element subspaces, the polynomial projection operators and the discrete forms which are exploited to construct the discrete schemes. In Section 4, the semi-discrete and fully-discrete schemes are introduced and the well posedness of the schemes are also discussed. A priori error estimates for the semi-discrete and the fully-discrete schemes are investigated in Section 5. The theoretical convergence rates are justified with four numerical examples in Section 6 . 


\section{Preliminaries AND THE MODEL PROBlem}

Let $\Omega \subset \mathbb{R}^{2}$ be a simply connected polygonal domain with boundary $\Gamma:=\partial \Omega$. We denote by $L^{2}(\Omega)$, the space of square integrable scalar functions with the standard inner product $(\psi, \phi)_{0, \Omega}:=\int_{\Omega} \psi \phi$. For each positive integer $s \in \mathbb{N}$, we define $H^{s}(\Omega)[2]$, the Sobolev space with standard norm

$$
\|\phi\|_{s, \Omega}:=\left(\sum_{0 \leq \alpha \leq s}\left\|\partial^{\alpha} \phi\right\|_{0, \Omega}^{2}\right)^{1 / 2}
$$

where $\alpha$ is multi index and $\partial^{\alpha} \phi$ denotes $\alpha$ th partial derivative of $\phi$. Let $t$ denote the time variable taking values in the interval $I:=(0, T]$, where $T$ is a given final time. Moreover, the function space $L^{2}\left(0, T ; H^{s}(\Omega)\right)$ consists of scalar functions $\phi$ such that for almost all $t \in[0, T], \phi(\cdot, t) \in H^{s}(\Omega)[15]$ with the norm,

$$
\|\phi\|_{L^{2}\left(0, T, H^{s}(\Omega)\right)}:=\left(\int_{0}^{T}\|\phi(t)\|_{s, \Omega}^{2}\right)^{1 / 2} ; \quad\|\phi\|_{L^{\infty}\left(0, T, H^{s}(\Omega)\right)}:=\underset{0 \leq t \leq T}{\operatorname{ess} \sup }\|\phi(t)\|_{s, \Omega} .
$$

In addition, given any Hilbert space $V$, we will denote by $[V]^{2}$ the space of vectors functions with entries in $V$ (see [2]). Further, we define $\partial_{t} \phi:=\frac{\mathrm{d} \phi}{\mathrm{d} t}, \partial_{t t} \phi:=\frac{\mathrm{d}^{2} \phi}{\mathrm{d} t^{2}}, \operatorname{div} \phi:=\frac{\mathrm{d} \phi}{\mathrm{d} x}+\frac{\mathrm{d} \phi}{\mathrm{d} y}, \operatorname{curl} \phi:=\left(\frac{\mathrm{d} \phi}{\mathrm{d} y},-\frac{\mathrm{d} \phi}{\mathrm{d} x}\right), \mathrm{D}^{2} \phi:=\left(\partial_{i j} \phi\right)_{1 \leq i, j \leq 2}$ denotes the Hessian matrix of $\phi$, and $\partial_{\boldsymbol{n}} \phi:=\nabla \phi \cdot \boldsymbol{n}$, where $\boldsymbol{n}$ is outward normal vector. For second order tensor fields $\boldsymbol{\sigma}, \boldsymbol{\tau}: \Omega \rightarrow \mathbb{R}^{2 \times 2}$, we define scalar product $:: \mathbb{R}^{2 \times 2} \times \mathbb{R}^{2 \times 2} \rightarrow \mathbb{R}$ by

$$
\boldsymbol{\sigma}: \boldsymbol{\tau}:=\sum_{1 \leq i, j \leq 2} \boldsymbol{\sigma}_{i j} \boldsymbol{\tau}_{i j}
$$

where $\boldsymbol{\sigma}_{i j}$ and $\boldsymbol{\tau}_{i j}$ are the entries at $(i, j)$-th position of $\boldsymbol{\sigma}$ and $\boldsymbol{\tau}$, respectively.

\subsection{Model problem}

We consider the time-dependent Navier-Stokes problem (for more details, see for instance [28,37]): given a sufficiently smooth force density $\mathbf{f} \in\left[L^{2}(\Omega)\right]^{2}$, we seek $(\boldsymbol{u}(t), p(t))$ such that:

$$
\begin{aligned}
\partial_{t} \boldsymbol{u}-\nu \boldsymbol{\Delta} \boldsymbol{u}+(\boldsymbol{u} \cdot \nabla) \boldsymbol{u}+\nabla p & =\mathbf{f} & & \text { in } \quad \Omega, \\
\operatorname{div} \boldsymbol{u} & =0 & & \text { in } \quad \Omega, \\
\boldsymbol{u} & =\mathbf{0} & & \text { on } \quad \Gamma, \\
(p, 1)_{0, \Omega} & =0, & & \\
\boldsymbol{u}(0) & =\boldsymbol{u}_{0}, & &
\end{aligned}
$$

where $\boldsymbol{u}, p$ are the velocity and the pressure fields, respectively, and $\nu>0$ is the viscosity of the fluid. We introduce the following Hilbert spaces:

$$
\mathbf{H}:=\left\{\boldsymbol{v} \in\left[H^{1}(\Omega)\right]^{2}: \boldsymbol{v}=\mathbf{0} \quad \text { on } \quad \Gamma\right\},
$$

and

$$
Q:=\left\{q \in L^{2}(\Omega):(q, 1)_{0, \Omega}=0\right\} .
$$

The standard velocity-pressure variational formulation of the Navier-Stokes problem reads as follows: find $(\boldsymbol{u}(t), p(t)) \in \mathbf{H} \times Q$, such that

$$
\begin{aligned}
\int_{\Omega} \partial_{t} \boldsymbol{u} \cdot \boldsymbol{v}+\nu \int_{\Omega} \nabla \boldsymbol{u}: \nabla \boldsymbol{v}+\int_{\Omega}(\boldsymbol{u} \cdot \nabla) \boldsymbol{u} \cdot \boldsymbol{v}-\int_{\Omega} p \operatorname{div} \boldsymbol{v}=\int_{\Omega} \mathbf{f} \cdot \boldsymbol{v} & \forall \boldsymbol{v} \in \mathbf{H}, \\
\int_{\Omega} q \operatorname{div} \boldsymbol{u}=0 & \forall q \in Q .
\end{aligned}
$$


It is well known that (2.2) admits a unique solution (see [28]). Let us introduce the following space of functions in $\mathbf{H}$ with vanishing divergence

$$
\mathbf{Z}:=\{\boldsymbol{v} \in \mathbf{H}: \operatorname{div} \boldsymbol{v}=0\} .
$$

Then, equation (2.2) can be rewritten in the following form: find $\boldsymbol{u}(t) \in \mathbf{Z}$ such that

$$
\int_{\Omega} \partial_{t} \boldsymbol{u} \cdot \boldsymbol{v}+\nu \int_{\Omega} \boldsymbol{\nabla} \boldsymbol{u}: \nabla \boldsymbol{v}+\int_{\Omega}(\boldsymbol{u} \cdot \nabla) \boldsymbol{u} \cdot \boldsymbol{v}=\int_{\Omega} \mathbf{f} \cdot \boldsymbol{v} \quad \forall \boldsymbol{v} \in \mathbf{Z} .
$$

Now, we reformulate the above problem as follows: since $\Omega$ is a simply connected domain, a well known result states that a vector function $\boldsymbol{v} \in \mathbf{Z}$ if and only if there exists a scalar function $\varphi \in H^{2}(\Omega$ ) (called stream-function) such that

$$
\boldsymbol{v}=\operatorname{curl} \varphi \in \mathbf{H} .
$$

The function $\varphi$ is defined up to a constant. Thus, we consider the following space

$$
H_{0}^{2}(\Omega):=\left\{\varphi \in H^{2}(\Omega): \varphi=\partial_{\boldsymbol{n}} \varphi=0 \text { on } \Gamma\right\} .
$$

We endow $H_{0}^{2}(\Omega)$ with the natural norm $\|\cdot\|_{2, \Omega}$. Then, $(2.2)$ can be formulated as follows: find $\psi(t) \in H_{0}^{2}(\Omega)$ such that

$$
\begin{aligned}
\int_{\Omega} \partial_{t}(\operatorname{curl} \psi) \cdot \operatorname{curl} \phi+\nu \int_{\Omega} \Delta \psi \Delta \phi+\int_{\Omega} \Delta \psi \operatorname{curl} \psi \cdot \nabla \phi & =\int_{\Omega} \mathbf{f} \cdot \operatorname{curl} \phi \quad \forall \phi \in H_{0}^{2}(\Omega), \\
\psi(0) & =\psi_{0} .
\end{aligned}
$$

Now, we introduce the following trilinear form as follows,

$$
\begin{aligned}
& C(\cdot ; \cdot, \cdot): H_{0}^{2}(\Omega) \times H_{0}^{2}(\Omega) \times H_{0}^{2}(\Omega) \rightarrow \mathbb{R}, \\
& C(v ; \psi, \phi):=\int_{\Omega} \Delta v \operatorname{curl} \psi \cdot \nabla \phi .
\end{aligned}
$$

It is observed that $C(v ; \phi, \phi)=0$ and $C(v ; \psi, \phi)=-C(v ; \phi, \psi)$. An application of Hölder inequality and the Sobolev's embedding theorem $H^{1}(\Omega) \hookrightarrow L^{4}(\Omega)$, we have

$$
C(v ; \psi, \phi) \leq C_{0}(\Omega)\|v\|_{2, \Omega}\|\psi\|_{2, \Omega}\|\phi\|_{2, \Omega} \quad \forall v, \psi, \phi \in H^{2}(\Omega) .
$$

Further, let $X^{\prime}$ be the dual space of $X$. For any smooth enough function $u, v \in H_{0}^{2}(\Omega)$, we define the function $\mathcal{G}(u, v) \in H^{-2}(\Omega)=\left[H_{0}^{2}(\Omega)\right]^{\prime}$ such that

$$
\langle\mathcal{G}(u, v), w\rangle_{-2,2, \Omega}:=C(u ; v, w) \quad \forall w \in H_{0}^{2}(\Omega),
$$

where $\langle\cdot, \cdot\rangle_{-2,2, \Omega}$ denotes duality pairing between $H^{-2}(\Omega)$ and $H_{0}^{2}(\Omega)$. It can be easily deduced that

$$
\|\mathcal{G}(u, u)\|_{-2, \Omega} \leq C_{0}(\Omega)\|u\|_{2, \Omega}^{2} \quad \forall u \in H_{0}^{2}(\Omega) .
$$

Since $\partial_{t}(\operatorname{curl} \psi)=\operatorname{curl}\left(\partial_{t} \psi\right)$, problem $(2.3)$ above can be written as follows: For a given function $\mathbf{f} \in$ $L^{2}\left(0, T ;\left[L^{2}(\Omega)\right]^{2}\right)$ and $\psi_{0} \in H_{0}^{2}(\Omega)$, the continuous weak formulation is defined as find $\psi \in L^{2}\left(0, T ; H_{0}^{2}(\Omega)\right)$ such that

$$
\begin{aligned}
A\left(\partial_{t} \psi, \phi\right)+\nu B(\psi, \phi)+C(\psi ; \psi, \phi) & =F(\phi) \quad \forall \phi \in H_{0}^{2}(\Omega), \\
\psi(0) & =\psi_{0},
\end{aligned}
$$

where $\psi(t) \in H_{0}^{2}(\Omega)$ is the stream-function of the velocity field $\boldsymbol{u}(t) \in \mathbf{Z}$ (i.e., $\boldsymbol{u}=\mathbf{c u r l} \psi$ ) and

$$
A(\cdot, \cdot): H_{0}^{2}(\Omega) \times H_{0}^{2}(\Omega) \rightarrow \mathbb{R}, \quad A(\psi, \phi):=\int_{\Omega} \operatorname{curl} \psi \cdot \operatorname{curl} \phi \quad \forall \psi, \phi \in H_{0}^{2}(\Omega),
$$




$$
\begin{array}{rlrl}
B(\cdot, \cdot): H_{0}^{2}(\Omega) \times H_{0}^{2}(\Omega) & \rightarrow \mathbb{R}, \quad B(\psi, \phi):=\int_{\Omega} \Delta \psi \Delta \phi & \forall \psi, \phi \in H_{0}^{2}(\Omega), \\
F(\cdot): H_{0}^{2}(\Omega) \rightarrow \mathbb{R}, \quad F(\phi):=\int_{\Omega} \mathbf{f} \cdot \operatorname{curl} \phi & \forall \phi \in H_{0}^{2}(\Omega) .
\end{array}
$$

Note that

$$
|B(\psi, \phi)| \leq\|\psi\|_{2, \Omega}\|\phi\|_{2, \Omega} \quad \forall \psi, \phi \in H_{0}^{2}(\Omega)
$$

and

$$
B(\phi, \phi) \geq C\|\phi\|_{2, \Omega}^{2} \quad \forall \phi \in H_{0}^{2}(\Omega)
$$

where $C$ is a positive generic constant. Next, we would like to discuss the well-posedness of continuous weak formulation (2.5).

Theorem 2.1. Let $\mathbf{f} \in L^{2}\left(0, T ;\left[L^{2}(\Omega)\right]^{2}\right)$ and $\psi_{0} \in H_{0}^{2}(\Omega)$. Then, there exists a unique solution $\psi \in$ $L^{2}\left(0, T ; H_{0}^{2}(\Omega)\right)$ of problem $(2.5)$.

Proof. For detail proof, we refer to Theorem 2.1 of [31] (see also [14]).

\section{ViRTUAL ELEMENT METhoD}

Let $\left\{\mathcal{T}_{h}\right\}_{h}$ be a sequence of decompositions of $\Omega$ into general polygonal elements $K$. Let $h_{K}$ denote the diameter of the element $K$ and $h$ the maximum of the diameters of all the elements of the mesh , i.e., $h:=$ $\max _{K \in \mathcal{T}_{h}} h_{K}$. In what follows, we denote by $N_{K}$ the number of vertices of $K$, by $e$ a generic edge of $\mathcal{T}_{h}$ and for all $e \in \partial K$, we define a unit normal vector $\boldsymbol{n}_{K}^{e}$ that points outside of $K$ and a unit tangent vector $\boldsymbol{t}_{K}^{e}$ to $K$. Further, we denote by $h_{e}$ the length of the edge $e$. For each vertex $V_{i}$, we associate a characteristic length $h_{V_{i}}$ which is the average of the diameter of the elements having $V_{i}$ as a vertex.

For the theoretical analysis, we will make the following assumptions: there exists a real number $C_{\mathcal{T}}>0$ such that, for every $h$ and every $K \in \mathcal{T}_{h}$,

\section{Assumption 3.1.}

(a) The ratio between the shortest edge and the diameter $h_{K}$ of $K$ is larger than $C_{\mathcal{T}}$;

(b) $K \in \mathcal{T}_{h}$ is star-shaped with respect to every point of a ball of radius $C_{\mathcal{T}} h_{K}$.

\subsection{Local and global virtual spaces}

Now, for any subset $\mathcal{D} \subseteq \mathbb{R}^{2}$ and non negative integer $k$, we will denote by $\mathcal{P}_{k}(\mathcal{D})$ the space of polynomials of degree up to $k$ defined on $\mathcal{D}$. Then, for $\left(s_{1}, s_{2}\right) \in \mathbb{N} \times \mathbb{N}$, we define the set of scale monomials as

$$
\mathcal{M}_{s}^{*}(K):=\left\{q^{*} \mid q^{*}=\left(\frac{x-x_{d}}{h_{K}}\right)^{s_{1}}\left(\frac{y-y_{d}}{h_{K}}\right)^{s_{2}} ; s=s_{1}+s_{2}\right\},
$$

where $\left(x_{d}, y_{d}\right)$ denotes centroid of $K$. Then, we define $\mathcal{M}_{s}(K):=\cup_{j \leq s} \mathcal{M}_{j}^{*}(K)$ as a basis of $\mathcal{P}_{s}(K)$. Analogously, we consider the set of the scaled monomials defined on each edge $e$ :

$$
\mathcal{M}_{s}(e):=\left\{1, \frac{\xi-\xi_{e}}{h_{e}},\left(\frac{\xi-\xi_{e}}{h_{e}}\right)^{2}, \ldots,\left(\frac{\xi-\xi_{e}}{h_{e}}\right)^{s}\right\}
$$

where $\xi_{e}$ is the midpoint of $e$.

Then, for any $k \geq 2$ and for every polygon $K \in \mathcal{T}_{h}$, we introduce the following preliminary local virtual space:

$$
\begin{aligned}
\widetilde{V}_{h}(K):= & \left\{\phi_{h} \in H^{2}(K): \Delta^{2} \phi_{h} \in \mathcal{P}_{k-2}(K),\left.\phi_{h}\right|_{\partial K} \in C^{0}(\partial K),\left.\phi_{h}\right|_{e} \in \mathcal{P}_{r}(e) \forall e \in \partial K,\right. \\
& \left.\left.\nabla \phi_{h}\right|_{\partial K} \in\left[C^{0}(\partial K)\right]^{2},\left.\partial_{n_{K}^{e}} \phi_{h}\right|_{e} \in \mathcal{P}_{\alpha}(e) \forall e \in \partial K\right\},
\end{aligned}
$$


where $r:=\max \{3, k\}$ and $\alpha:=k-1$.

Next, for a given $\phi_{h} \in \widetilde{V}_{h}(K)$, we introduce five sets $\mathbf{D}_{\mathbf{1}}-\mathbf{D}_{\mathbf{5}}$ of linear operators from the local virtual space $\widetilde{V}_{h}(K)$ into $\mathbb{R}$.

- $\mathbf{D}_{\mathbf{1}}$ : contains linear operators evaluating $\phi_{h}$ at the $N_{K}$ vertices of $K$;

- $\mathbf{D}_{2}$ : contains linear operators evaluating $h_{V_{i}} \nabla \phi_{h}\left(V_{i}\right)$ for all vertices $V_{i}$ of $K$, where $1 \leq i \leq N_{K}$;

- $\mathbf{D}_{\mathbf{3}}$ : for $r>3$, the moments $\frac{1}{h_{e}} \int_{e} q(\zeta) \phi_{h}(\zeta) \mathrm{d} \zeta \quad \forall q \in \mathcal{M}_{r-4}(e), \quad \forall$ edge $e$;

- $\mathbf{D}_{4}$ : for $\alpha>1$, the moments $\int_{e} q(\zeta) \partial_{\boldsymbol{n}_{K}^{e}} \phi_{h}(\zeta) \mathrm{d} \zeta \quad \forall q \in \mathcal{M}_{\alpha-2}(e), \quad \forall$ edge $e$;

- $\mathbf{D}_{\mathbf{5}}$ : for $k \geq 4$, the moments $\frac{1}{h_{K}^{2}} \int_{K} q(\boldsymbol{x}) \phi_{h}(\boldsymbol{x}) \mathrm{d} \boldsymbol{x} \quad \forall q \in \mathcal{M}_{k-4}(K), \quad \forall$ polygon $K$.

In order to construct the discrete scheme, we first observe that

$$
\int_{\Omega} \Delta \varphi \Delta \phi=\int_{\Omega} \mathrm{D}^{2} \varphi: \mathrm{D}^{2} \phi
$$

Then, we decompose the bilinear form (2.7) in the following element by element contribution:

$$
B(\varphi, \phi)=\int_{\Omega} \mathrm{D}^{2} \varphi: \mathrm{D}^{2} \phi=\sum_{K \in \mathcal{T}_{h}} B_{K}(\varphi, \phi)=\sum_{K \in \mathcal{T}_{h}} \int_{K} \mathrm{D}^{2} \varphi: \mathrm{D}^{2} \phi, \quad \forall \varphi, \phi \in H_{0}^{2}(\Omega) .
$$

In what follows, we are going to build the discrete version of the local bilinear forms listed above. With this aim, we define the following projector operator $\Pi_{K}^{k, \Delta}: \widetilde{V}_{h}(K) \longrightarrow \mathcal{P}_{k}(K) \subseteq \widetilde{V}_{h}(K)$ for each $\phi_{h} \in \widetilde{V}_{h}(K)$, as the solution of the local problems (on each polygon $K$ ):

$$
\begin{aligned}
B_{K}\left(\Pi_{K}^{k, \Delta} \phi_{h}, q\right) & =B_{K}\left(\phi_{h}, q\right) \quad \forall q \in \mathcal{P}_{k}(K), \\
\widehat{\Pi_{K}^{k, \Delta} \phi_{h}} & =\widehat{\phi_{h}}, \quad \widehat{\nabla \Pi_{K}^{k, \Delta} \phi_{h}}=\widehat{\nabla \phi_{h}},
\end{aligned}
$$

where $\widehat{(\cdot)}$ is defined as follows:

$$
\widehat{\chi_{h}}:=\frac{1}{N_{K}} \sum_{i=1}^{N_{K}} \chi_{h}\left(V_{i}\right) \quad \forall \chi_{h} \in C^{0}(\partial K),
$$

and $V_{i}, 1 \leq i \leq N_{K}$, are the vertices of $K$.

The following result establishes that the projector $\Pi_{K}^{k, \Delta}$ is computable using the output values of the sets $\mathbf{D}_{1}-\mathbf{D}_{5}$.

Lemma 3.2. The operator $\Pi_{K}^{k, \Delta}: \widetilde{V}_{h}(K) \longrightarrow \mathcal{P}_{k}(K)$ is explicitly computable for every $\phi_{h} \in \widetilde{V}_{h}(K)$, using only the information of the linear operators $\mathbf{D}_{\mathbf{1}}-\mathbf{D}_{\mathbf{5}}$.

Proof. For detail proof, we refer to [21].

For each $k \geq 2$ and for any $K \in \mathcal{T}_{h}$ our local enhanced virtual space is given by:

$$
W_{h}^{k}(K):=\left\{\phi_{h} \in \widetilde{V}_{h}(K): \int_{K} q^{*} \Pi_{K}^{k, \Delta} \phi_{h}=\int_{K} q^{*} \phi_{h}, \quad \forall q^{*} \in \mathcal{M}_{k-2}^{*}(K) \cup \mathcal{M}_{k-3}^{*}(K)\right\},
$$

where $\mathcal{M}_{k-2}^{*}(K)$ and $\mathcal{M}_{k-3}^{*}(K)$ are scaled monomials of degree $k-2$ and $k-3$, respectively, with the convention that $\mathcal{M}_{-1}^{*}(K)=\emptyset$.

By using the linear operators $\mathbf{D}_{\mathbf{1}}-\mathbf{D}_{\mathbf{5}}$, we can evaluate $\Pi_{K}^{k, \Delta} \phi_{h}$ for all $\phi_{h} \in W_{h}^{k}(K)$, which is stated explicitly in the next result. 
Lemma 3.3. The operator $\Pi_{K}^{k, \Delta}$ is well defined and computable on the local enhanced virtual space $W_{h}^{k}(K)$.

Proof. From definition of local enhanced virtual space (3.3), we deduce that $W_{h}^{k}(K) \subseteq \widetilde{V}_{h}(K)$. Consequently, for $\phi_{h} \in W_{h}^{k}(K)$ implies $\phi_{h} \in \widetilde{V}_{h}(K)$ and hence $\Pi_{K}^{k, \Delta} \phi_{h}$ is well-defined and computable.

On the other hand, we observe that $\mathcal{P}_{k}(K) \subseteq W_{h}^{k}(K)$ which will guarantee the good approximation properties for the space. Moreover, following similar arguments presented in [3,16] (see also [21]) we obtain that the sets of linear operators $\mathbf{D}_{\mathbf{1}}-\mathbf{D}_{\mathbf{5}}$ constitutes a set of degrees of freedom for $W_{h}^{k}(K)$.

Now, we introduce the global virtual space by combining the local spaces $W_{h}^{k}(K)$ and incorporating the homogeneous Dirichlet boundary conditions. For every decomposition $\mathcal{T}_{h}$ of $\Omega$ into polygons $K$, we define

$$
W_{h}:=\left\{\phi_{h} \in H_{0}^{2}(\Omega):\left.\phi_{h}\right|_{K} \in W_{h}^{k}(K)\right\} .
$$

\subsection{Construction of bilinear forms and the force term}

In order to build the discrete local and global forms, we observe that the particular condition appearing in the definition of the local virtual space $W_{h}^{k}(K)$ will be useful to construct an $L^{2}$-projection which will be employed to build the discrete bilinear forms. In particular, we consider the $L^{2}(K)$-projection onto $\mathcal{P}_{k-2}(K)$. For each $\phi \in L^{2}(K), \Pi_{K}^{k-2} \phi \in \mathcal{P}_{k-2}(K)$ satisfies

$$
\int_{K}\left(\Pi_{K}^{k-2} \phi\right) q=\int_{K} \phi q \quad \forall q \in \mathcal{P}_{k-2}(K) .
$$

The following lemma establishes that $\Pi_{K}^{k-2}$ is computable on $W_{h}^{k}(K)$. The proof follows from the definition of the local virtual space and the set of degrees of freedom.

Lemma 3.4. The operator $\Pi_{K}^{k-2}: W_{h}^{k}(K) \longrightarrow \mathcal{P}_{k-2}(K)$ is explicitly computable for each $\phi_{h} \in W_{h}^{k}(K)$, using only the information of the set of degrees freedom $\mathbf{D}_{\mathbf{1}}-\mathbf{D}_{\mathbf{5}}$.

Proof. For a detail proof, we refer to [21].

Now, for $k \geq 2$, we will introduce some additional projectors which will be used to write the virtual scheme. First, we define $\Pi_{K}^{k, \nabla^{\perp}}: W_{h}^{k}(K) \longrightarrow \mathcal{P}_{k}(K) \subseteq W_{h}^{k}(K)$ for each $\phi_{h} \in W_{h}^{k}(K)$ as the solution of the following local problem.

$$
\begin{aligned}
\int_{K} \operatorname{curl} \Pi_{K}^{k, \nabla^{\perp}} \phi_{h} \cdot \operatorname{curl} q & =\int_{K} \operatorname{curl} \phi_{h} \cdot \operatorname{curl} q \quad \forall q \in \mathcal{P}_{k}(K), \\
\Pi_{K}^{\widehat{k, \nabla^{\perp}}} \phi_{h} & =\widehat{\phi_{h}},
\end{aligned}
$$

where $\widehat{(\cdot)}$ has been defined in (3.2). The following result states that this operator is fully computable.

Lemma 3.5. The operator $\Pi_{K}^{k, \nabla^{\perp}}: W_{h}^{k}(K) \longrightarrow \mathcal{P}_{k}(K) \subseteq W_{h}^{k}(K)$ is explicitly computable for each $\phi_{h} \in$ $W_{h}^{k}(K)$, using only the information of the set of degrees freedom $\mathbf{D}_{\mathbf{1}}-\mathbf{D}_{\mathbf{5}}$.

Proof. First we note that (3.5b) is computable using the information of the set $\mathbf{D}_{\mathbf{1}}$. On the other hand, we integrate by parts on the right hand side of $(3.5 \mathrm{a})$ to obtain:

$$
\begin{aligned}
\int_{K} \operatorname{curl} \phi_{h} \cdot \operatorname{curl} q & =-\int_{K} \phi_{h} \Delta q+\int_{\partial K} \phi_{h} \partial_{\boldsymbol{n}_{K}} q & & \forall q \in \mathcal{P}_{k}(K) \\
& =-\int_{K} \Pi_{K}^{k-2} \phi_{h} \Delta q+\int_{\partial K} \phi_{h} \partial_{\boldsymbol{n}_{K}} q & & \forall q \in \mathcal{P}_{k}(K),
\end{aligned}
$$

where we have used the fact that $\Delta q \in \mathcal{P}_{k-2}(K)$ and the definition of the projection $\Pi_{K}^{k-2}(c f$. (3.4)). The previous equality allows us to conclude that the polynomial $\Pi_{K}^{k, \nabla^{\perp}} \phi_{h}$ can be explicitly computed from the degrees of freedom $\mathbf{D}_{\mathbf{1}}-\mathbf{D}_{\mathbf{5}}$. 
Now, we will introduce an additional projection operator onto the polynomial space $\left[\mathcal{P}_{k-1}(K)\right]^{2}$, which will be used to construct a local approximation of $A(\cdot, \cdot)$ and $C(\cdot ; \cdot, \cdot)$. For $K \in \mathcal{T}_{h}$, and $\boldsymbol{v} \in\left[L^{2}(K)\right]^{2}$, we define $\Pi_{K}^{k-1}:\left[L^{2}(K)\right]^{2} \rightarrow\left[\mathcal{P}_{k-1}(K)\right]^{2}$ by

$$
\int_{K} \boldsymbol{\Pi}_{K}^{k-1} \boldsymbol{v} \cdot \mathbf{q}=\int_{K} \boldsymbol{v} \cdot \mathbf{q} \quad \forall \mathbf{q} \in\left[\mathcal{P}_{k-1}(K)\right]^{2} .
$$

We observe that for any $\phi_{h} \in W_{h}^{k}(K)$, the vector functions $\boldsymbol{\Pi}_{K}^{k-1} \operatorname{curl} \phi_{h} \in\left[\mathcal{P}_{k-1}(K)\right]^{2}$ and $\Pi_{K}^{k-1} \nabla \phi_{h} \in$ $\left[\mathcal{P}_{k-1}(K)\right]^{2}$ can be explicitly computed from the degrees of freedom $\mathbf{D}_{\mathbf{1}}-\mathbf{D}_{\mathbf{5}}$. In fact, for all $K \in \mathcal{T}_{h}$ and for all $\phi_{h} \in W_{h}^{k}(K)$, using integration by parts on the right-hand side of (3.6) (with curl $\phi_{h}$ instead of $\boldsymbol{v}$ ), we have (see [28])

$$
\begin{aligned}
\int_{K} \operatorname{curl} \phi_{h} \cdot \mathbf{q} & =\int_{K} \phi_{h} \operatorname{rot} \mathbf{q}-\int_{\partial K} \phi_{h}\left(\mathbf{q} \cdot \boldsymbol{t}_{K}\right) & & \forall \mathbf{q} \in\left[\mathcal{P}_{k-1}(K)\right]^{2} \\
& =\int_{K}\left(\Pi_{K}^{k-2} \phi_{h}\right) \operatorname{rot} \mathbf{q}-\int_{\partial K} \phi_{h}\left(\mathbf{q} \cdot \boldsymbol{t}_{K}\right) & & \forall \mathbf{q} \in\left[\mathcal{P}_{k-1}(K)\right]^{2},
\end{aligned}
$$

where we have used (3.4). The first term on the right-hand side above depends only on $\Pi_{K}^{k-2} \phi_{h}$ and this depends only on the values of the degrees of freedom (see Lem. 3.4). The second term is an integral on the boundary of the element $K$, which is fully computable.

Next, we use the above projection operators to construct computable approximations of the continuous bilinear and trilinear forms, and for the right-hand side. First, let $s_{K}^{\Delta}(\cdot, \cdot)$ and $s_{K}^{\text {curl }}(\cdot, \cdot)$ be any symmetric positive definite bilinear forms to be chosen as to satisfy:

$$
\begin{array}{llll}
c_{0} B_{K}\left(\phi_{h}, \phi_{h}\right) \leq s_{K}^{\Delta}\left(\phi_{h}, \phi_{h}\right) \leq c_{1} B_{K}\left(\phi_{h}, \phi_{h}\right) & \forall \phi_{h} \in W_{h}^{k}(K), & \text { with } \quad \Pi_{K}^{k, \Delta} \phi_{h}=0, \\
c_{2} A_{K}\left(\phi_{h}, \phi_{h}\right) \leq s_{K}^{\text {curl }}\left(\phi_{h}, \phi_{h}\right) \leq c_{3} A_{K}\left(\phi_{h}, \phi_{h}\right) & \forall \phi_{h} \in W_{h}^{k}(K), \quad \text { with } \quad \Pi_{K}^{k, \nabla^{\perp}} \phi_{h}=0,
\end{array}
$$

with $c_{0}, c_{1}, c_{2}$ and $c_{3}$ are positive constants independent of $h$ and $K$. From (3.7), we deduce that $s_{K}^{\Delta}(\cdot, \cdot)$, and $s_{K}^{\text {curl }}(\cdot, \cdot)$ scale same as $B_{K}(\cdot, \cdot)$ and $A_{K}(\cdot, \cdot)$, respectively.

On each element $K$, we define the local discrete bilinear forms

$$
A_{K}^{h}(\cdot, \cdot): W_{h}^{k}(K) \times W_{h}^{k}(K) \rightarrow \mathbb{R}, \quad B_{K}^{h}(\cdot, \cdot): W_{h}^{k}(K) \times W_{h}^{k}(K) \rightarrow \mathbb{R}
$$

as follow, for all $\psi_{h}, \phi_{h} \in W_{h}^{k}(K)$

$$
\begin{aligned}
& A_{K}^{h}\left(\psi_{h}, \phi_{h}\right):=A_{K}\left(\Pi_{K}^{k, \nabla^{\perp}} \psi_{h}, \Pi_{K}^{k, \nabla^{\perp}} \phi_{h}\right)+s_{K}^{\operatorname{curl}}\left(\psi_{h}-\Pi_{K}^{k, \nabla^{\perp}} \psi_{h}, \phi_{h}-\Pi_{K}^{k, \nabla^{\perp}} \phi_{h}\right), \\
& B_{K}^{h}\left(\psi_{h}, \phi_{h}\right):=B_{K}\left(\Pi_{K}^{k, \Delta} \psi_{h}, \Pi_{K}^{k, \Delta} \phi_{h}\right)+s_{K}^{\Delta}\left(\psi_{h}-\Pi_{K}^{k, \Delta} \psi_{h}, \phi_{h}-\Pi_{K}^{k, \Delta} \phi_{h}\right) .
\end{aligned}
$$

It can be observed that the forms $s_{K}^{\text {curl }}(\cdot, \cdot)$ and $s_{K}^{\Delta}(\cdot, \cdot)$ reduce to zero when one of the two entries $\phi_{h}$ or $\psi_{h}$ is a polynomial function. Different computable form of the stabilizers are available in the literature $[5,16,36]$. However, we choose the following representation

$$
\begin{gathered}
s_{K}^{\text {curl }}\left(\phi_{i}-\Pi_{K}^{k, \nabla^{\perp}} \phi_{i}, \phi_{j}-\Pi_{K}^{k, \nabla^{\perp}} \phi_{j}\right):=\alpha_{K}^{\text {curl }} \sum_{z=1}^{N_{K}^{\text {dof }}} \operatorname{dof}_{z}\left(\left(I-\Pi_{K}^{k, \nabla^{\perp}}\right) \phi_{i}\right) \operatorname{dof}_{z}\left(\left(I-\Pi_{K}^{k, \nabla^{\perp}}\right) \phi_{j}\right), \\
s_{K}^{\Delta}\left(\phi_{i}-\Pi_{K}^{k, \Delta} \phi_{i}, \phi_{j}-\Pi_{K}^{k, \Delta} \phi_{j}\right):=\alpha_{K}^{\Delta} \sum_{z=1}^{N_{K}^{\text {dof }}} \operatorname{dof}_{z}\left(\left(I-\Pi_{K}^{k, \Delta}\right) \phi_{i}\right) \operatorname{dof}_{z}\left(\left(I-\Pi_{K}^{k, \Delta}\right) \phi_{j}\right),
\end{gathered}
$$


where $\alpha_{K}^{\text {curl }}$ and $\alpha_{K}^{\Delta}$ are the suitable constants, and $N_{K}^{\text {dof }}$ denotes the number of degrees freedom of $W_{h}^{k}(K)$. Adding the local contribution, the global forms are defined as

$$
\begin{array}{ll}
A^{h}\left(\psi_{h}, \phi_{h}\right):=\sum_{K \in \mathcal{T}_{h}} A_{K}^{h}\left(\psi_{h}, \phi_{h}\right) & \forall \psi_{h}, \phi_{h} \in W_{h}, \\
B^{h}\left(\psi_{h}, \phi_{h}\right):=\sum_{K \in \mathcal{T}_{h}} B_{K}^{h}\left(\psi_{h}, \phi_{h}\right) & \forall \psi_{h}, \phi_{h} \in W_{h} .
\end{array}
$$

The following result establishes the usual consistency and stability properties for the discrete local bilinear forms.

Proposition 3.6. For $k \geq 2$, the local bilinear forms $A_{K}^{h}(\cdot, \cdot)$ and $B_{K}^{h}(\cdot, \cdot)$ on each element $K$ satisfy

- Consistency: for all $h>0$ and for all $K \in \mathcal{T}_{h}$, we have that

$$
\begin{array}{lll}
A_{K}^{h}\left(q, \phi_{h}\right)=A_{K}\left(q, \phi_{h}\right) & \forall q \in \mathcal{P}_{k}(K), & \forall \phi_{h} \in W_{h}^{k}(K), \\
B_{K}^{h}\left(q, \phi_{h}\right)=B_{K}\left(q, \phi_{h}\right) & \forall q \in \mathcal{P}_{k}(K), & \forall \phi_{h} \in W_{h}^{k}(K) .
\end{array}
$$

- Stability and boundedness: There exist positive constants $\alpha_{i}, i=1, \ldots, 4$, independent of $K$, such that:

$$
\begin{array}{ll}
\alpha_{1} A_{K}\left(\phi_{h}, \phi_{h}\right) \leq A_{K}^{h}\left(\phi_{h}, \phi_{h}\right) \leq \alpha_{2} A_{K}\left(\phi_{h}, \phi_{h}\right) & \forall \phi_{h} \in W_{h}^{k}(K), \\
\alpha_{3} B_{K}\left(\phi_{h}, \phi_{h}\right) \leq B_{K}^{h}\left(\phi_{h}, \phi_{h}\right) \leq \alpha_{4} B_{K}\left(\phi_{h}, \phi_{h}\right) & \forall \phi_{h} \in W_{h}^{k}(K) .
\end{array}
$$

Proof. For further details, we refer to [19,21,35].

We observe that from the symmetry of $A^{h}(\cdot, \cdot)$ and $B^{h}(\cdot, \cdot)$ and the stability conditions stated before imply the continuity of $A^{h}$ and $B^{h}$. In fact, for all $\psi_{h}, \phi_{h} \in W_{h}$ :

$$
\begin{aligned}
& \left|A^{h}\left(\psi_{h}, \phi_{h}\right)\right| \leq C_{A}\left\|\psi_{h}\right\|_{1, \Omega}\left\|\phi_{h}\right\|_{1, \Omega}, \\
& \left|B^{h}\left(\psi_{h}, \phi_{h}\right)\right| \leq C_{B}\left\|\psi_{h}\right\|_{2, \Omega}\left\|\phi_{h}\right\|_{2, \Omega} .
\end{aligned}
$$

The following result establishes that by virtue of $(3.13)$, bilinear form $B^{h}(\cdot, \cdot)$ is uniformly elliptic.

Lemma 3.7. There exists a constant $\alpha>0$, independent of $h$, such that

$$
B^{h}\left(v_{h}, v_{h}\right) \geq \alpha\left\|v_{h}\right\|_{2, \Omega}^{2} \quad \forall v_{h} \in W_{h} .
$$

Now, we proceed to discretize the force function as follows

$$
F_{K}^{h}(\cdot): W_{h}^{k}(K) \rightarrow \mathbb{R}
$$

such that

$$
F_{K}^{h}\left(\phi_{h}\right):=\int_{K} \boldsymbol{\Pi}_{K}^{k-1} \mathbf{f} \cdot \operatorname{curl} \phi_{h}=\int_{K} \mathbf{f} \cdot \boldsymbol{\Pi}_{K}^{k-1} \operatorname{curl} \phi_{h} \quad \forall \phi_{h} \in W_{h}^{k}(K) .
$$

Globally, the force function $F_{K}^{h}$ is defined as follows

$$
F^{h}\left(\phi_{h}\right):=\sum_{K \in \mathcal{T}_{h}} F_{K}^{h}\left(\phi_{h}\right) \quad \forall \phi_{h} \in W_{h}
$$




\subsection{Discretization of the nonlinear term}

In this section, we would like to discretize the trilinear term associated with the problem (2.3). Using the projection operators $\Pi_{K}^{k, \Delta}$ and $\Pi_{K}^{k-1}$, we discretize the trilinear term.

$$
C_{K}^{h}(\cdot ; \cdot, \cdot): W_{h}^{k}(K) \times W_{h}^{k}(K) \times W_{h}^{k}(K) \rightarrow \mathbb{R}
$$

such that

$$
C_{K}^{h}\left(v_{h} ; \psi_{h}, \phi_{h}\right):=\int_{K}\left(\Delta \Pi_{K}^{k, \Delta} v_{h}\right) \Pi_{K}^{k-1} \operatorname{curl} \psi_{h} \cdot \Pi_{K}^{k-1} \nabla \phi_{h} \quad \forall v_{h}, \psi_{h}, \phi_{h} \in W_{h}^{k}(K) .
$$

The term is fully computable from the degrees of freedom. Globally, the trilinear term is defined as

$$
C^{h}\left(v_{h} ; \psi_{h}, \phi_{h}\right):=\sum_{K \in \mathcal{T}_{h}} C_{K}^{h}\left(v_{h} ; \psi_{h}, \phi_{h}\right) \quad \forall v_{h}, \psi_{h}, \phi_{h} \in W_{h} .
$$

Moreover, it can be shown that the discrete trilinear form $C^{h}(\cdot ; \cdot, \cdot)$ is uniformly bounded on $W_{h}$.

Lemma 3.8. Let $C^{h}\left(v_{h} ; \psi_{h}, \phi_{h}\right)$ be the trilinear form defined in (3.17). Then there exists a positive constant $\hat{C}$ such that

$$
\left|C^{h}\left(v_{h} ; \psi_{h}, \phi_{h}\right)\right| \leq \hat{C}\left\|v_{h}\right\|_{2, \Omega}\left\|\psi_{h}\right\|_{2, \Omega}\left\|\phi_{h}\right\|_{2, \Omega},
$$

where $\hat{C}$ is independent of mesh size $h$.

Proof. An application of boundedness of the projection operators $\Pi_{K}^{k, \Delta}$ and $\Pi_{K}^{k-1}$, Hölder inequality, and Sobolev's embedding theorem yields the proof.

Moreover, we have the following properties of $C^{h}(\cdot ; \cdot, \cdot)$

$$
C^{h}\left(v_{h} ; \phi_{h}, \phi_{h}\right)=0 \quad \forall v_{h}, \phi_{h} \in W_{h}
$$

In addition, we observe that $C^{h}(\cdot ; \cdot, \cdot)$ can be extended to $H_{0}^{2}(\Omega)$ only taking the projections of the continuous $v, \psi, \phi \in H_{0}^{2}(\Omega)$.

Remark 3.9. The discrete trilinear form $C^{h}(\cdot ; \cdot, \cdot)$ does not contain non-polynomial part or stabilizer. It is defined using the projection operators $\Pi_{K}^{k, \Delta}$ and $\Pi_{K}^{k-1}$ which are computable from the information provided by the degrees of freedom. With this definition, we will show that the semi-discrete and fully-discrete schemes are well-posed and we will obtain the corresponding error estimates.

\section{DisCRETE SCHEMES AND THEIR WELL POSEDNESS}

In this section, we will introduce the semi-discrete and fully-discrete virtual element schemes for problem (2.5), by using the discrete forms introduced in Sections 3.2 and 3.3. We will also prove that under some assumptions on $\nu$, the fully-discrete scheme is well posed.

\subsection{Semi-discrete formulation}

The semi-discrete VEM formulation for the time dependent Navier-Stokes problem reads as follows. For all $t>0$, find $\psi_{h} \in L^{2}\left(0, T ; W_{h}\right)$ such that

$$
A^{h}\left(\partial_{t} \psi_{h}(t), \phi_{h}\right)+\nu B^{h}\left(\psi_{h}(t), \phi_{h}\right)+C^{h}\left(\psi_{h}(t) ; \psi_{h}(t), \phi_{h}\right)=F^{h}\left(\phi_{h}\right) \quad \forall \phi_{h} \in W_{h} .
$$

Additionally, we set $\psi_{h}(0)=\psi_{I}(0)$, where $\psi_{I}(0)$ is a suitable interpolation of $\psi_{0}$ (see Prop. 5.2). In this section, we will discuss the well-posedness of semi-discrete scheme (4.1). 
First, we observe that the matrix representation corresponding to the discrete bilinear form $A^{h}(\cdot, \cdot)$ is positive definite and hence inverse exists. Further, let us assume that $\mathbf{A}, \mathbf{B}$ be the matrix representation corresponding to the discrete forms $A^{h}(\cdot, \cdot), B^{h}(\cdot, \cdot)$, respectively. Therefore, problem (4.1) reduces to a system of nonlinear differential equations as follows

$$
\begin{aligned}
\mathbf{A} \frac{\mathrm{d} \boldsymbol{\psi}_{h}}{\mathrm{~d} t}+\nu \mathbf{B} \boldsymbol{\psi}_{h}+\mathbf{C}\left(\boldsymbol{\psi}_{h}\right) & =\mathbf{F} \\
\boldsymbol{\psi}_{h}(0) & =\boldsymbol{\psi}_{0},
\end{aligned}
$$

where $\boldsymbol{\psi}_{h}$ denotes the vector whose entries are the components in the basis of $\psi_{h}$. Moreover, $\mathbf{C}\left(\boldsymbol{\psi}_{h}\right)$ is the matrix corresponding to the nonlinear term and $\mathbf{F}$ be the right hand side load vector corresponding to the basis $\phi_{h}$. Before going into further details, we would like to prove that the nonlinear term , i.e., $C^{h}\left(\psi_{h} ; \psi_{h}, \phi_{h}\right)$ satisfies Lipschitz's continuity condition. In this direction, let $\psi_{h}^{1}, \psi_{h}^{2}$ be two elements in $W_{h}$. Then, we can write as

$$
\begin{aligned}
\left|C^{h}\left(\psi_{h}^{1} ; \psi_{h}^{1}, \phi_{h}\right)-C^{h}\left(\psi_{h}^{2} ; \psi_{h}^{2}, \phi_{h}\right)\right|= & \left|C^{h}\left(\psi_{h}^{1} ; \psi_{h}^{1}, \phi_{h}\right)-C\left(\psi_{h}^{2} ; \psi_{h}^{1}, \phi_{h}\right)+C\left(\psi_{h}^{2} ; \psi_{h}^{1}, \phi_{h}\right)-C^{h}\left(\psi_{h}^{2} ; \psi_{h}^{2}, \phi_{h}\right)\right| \\
\leq & \sum_{K \in \mathcal{T}_{h}}(\underbrace{\left|\int_{K} \Delta \Pi_{K}^{k, \Delta}\left(\psi_{h}^{1}-\psi_{h}^{2}\right) \Pi_{K}^{k-1} \operatorname{curl} \psi_{h}^{1} \cdot \boldsymbol{\Pi}_{K}^{k-1} \nabla \phi_{h}\right|}_{:=L_{1}} \\
& +\underbrace{\mid \int_{K} \Delta \Pi_{K}^{k, \Delta} \psi_{h}^{2} \Pi_{K}^{k-1} \operatorname{curl}\left(\psi_{h}^{1}-\psi_{h}^{2}\right) \cdot \Pi_{K}^{k-1} \nabla \phi_{h}}_{:=L_{2}} \mid) .
\end{aligned}
$$

An application of Hölder inequality and using the continuity of $\Pi_{K}^{k-1}$ with respect to $L^{4}$-norm and stability of $\Pi_{K}^{k, \Delta}$, we obtain

$$
\sum_{K \in \mathcal{T}_{h}} L_{1} \leq C\left\|\psi_{h}^{1}-\psi_{h}^{2}\right\|_{2, \Omega}\left\|\operatorname{curl} \psi_{h}^{1}\right\|_{L^{4}(\Omega)}\left\|\nabla \phi_{h}\right\|_{L^{4}(\Omega)},
$$

using Sobolev's embedding theorem, we obtain

$$
\sum_{K \in \mathcal{T}_{h}} L_{1} \leq C\left\|\psi_{h}^{1}-\psi_{h}^{2}\right\|_{2, \Omega}\left\|\operatorname{curl} \psi_{h}^{1}\right\|_{1, \Omega}\left\|\nabla \phi_{h}\right\|_{1, \Omega}
$$

Using analogous arguments, we derive that

$$
\sum_{K \in \mathcal{T}_{h}} L_{2} \leq C\left\|\psi_{h}^{2}\right\|_{2, \Omega}\left\|\operatorname{curl}\left(\psi_{h}^{1}-\psi_{h}^{2}\right)\right\|_{1, \Omega}\left\|\nabla \phi_{h}\right\|_{1, \Omega}
$$

Inserting (4.5) and (4.6) into (4.4), we can claim that the nonlinear term $C^{h}\left(\psi_{h} ; \psi_{h}, \phi_{h}\right)$ is Lipschitz continuous. Therefore, from Picard's Theorem of existence and uniqueness of system of differential equation, we can deduce that (4.2) and (4.3) has a unique solution.

\subsection{Fully-discrete formulation}

A classical backward Euler integration method is employed for the time discretization of (4.1) with time step $\Delta t=T / N$, where $N$ is a positive integer. In addition, we introduce $\psi_{h}^{n}:=\psi_{h}\left(t_{n}\right)$ for $n=0,1,2, \ldots, N$. This results in the following fully discrete method: find $\psi_{h}^{n} \in W_{h}$ such that

$$
\begin{aligned}
A^{h}\left(\frac{\psi_{h}^{n}-\psi_{h}^{n-1}}{\Delta t}, \phi_{h}\right)+\nu B^{h}\left(\psi_{h}^{n}, \phi_{h}\right)+C^{h}\left(\psi_{h}^{n} ; \psi_{h}^{n}, \phi_{h}\right) & =F^{h}\left(\phi_{h}\right) \quad \forall \phi_{h} \in W_{h}, \\
\psi_{h}^{0} & =\psi_{I}(0),
\end{aligned}
$$


where $\psi_{h}^{0} \in W_{h}$ is an initial approximation of $\psi$ at $t=0$. Next, we prove the well posedness of the fullydiscrete scheme (4.7). In this direction, we first recollect Brouwer's fixed point theorem. Then, under certain assumption, we will show that the fully-discrete scheme has unique solution $\psi_{h}^{n}$ and the solution is bounded, i.e., $\left\|\psi_{h}^{n}\right\|_{2, \Omega} \leq \mathcal{R}$, where $\mathcal{R}$ is a positive constant which will be specified later.

Theorem 4.1 (Brouwer's theorem). Let $\mathcal{K}$ be a Banach space and let $\mathcal{B} \subset \mathcal{K}$ be a compact and convex subset. If $\mathcal{L}: \mathcal{B} \rightarrow \mathcal{B}$ is continuous, then $\mathcal{L}$ has a fixed point.

Theorem 4.2. Assume that

$$
\max \left\{\frac{2 \hat{C}^{2}}{\alpha_{3}^{2} \nu^{2}}, \frac{2 \hat{C}^{2} C_{B}^{2}}{\alpha_{3}^{4} \nu^{2}}\right\}\left\|\psi_{h}\right\|_{L^{\infty}\left(0, t_{n-1} ; H^{2}(\Omega)\right)}^{2}<1 .
$$

Then, for $1 \leq n \leq N$ and for sufficiently small values of $\Delta t$, there exists a unique solution of the fully-discrete problem (4.7) and the solution $\psi_{h}^{n}$ satisfies the condition $\left\|\psi_{h}^{n}\right\|_{2, \Omega} \leq \mathcal{R}$, with

$$
\mathcal{R}:=\frac{\left(\frac{\Delta t}{\alpha_{1} \alpha_{3} \nu}\|\mathbf{f}\|_{L^{\infty}\left(0, t_{n-1} ; L^{2}(\Omega)\right)}^{2}+\frac{2 C_{B}^{2}}{\alpha_{3}^{2}}\left\|\psi_{h}\right\|_{L^{\infty}\left(0, t_{n-1} ; H^{2}(\Omega)\right)}^{2}\right)^{1 / 2}}{\left(1-\frac{2 \hat{C}^{2}}{\alpha_{3}^{2} \nu^{2}}\left\|\psi_{h}\right\|_{L^{\infty}\left(0, t_{n-1} ; H^{2}(\Omega)\right)}^{2}\right)^{1 / 2}} .
$$

Proof. Let $\psi_{h}^{n-1} \in W_{h}$. Define a mapping $\mathcal{F}: W_{h} \rightarrow W_{h}$ such that $\psi_{h}^{n}=\mathcal{F}\left(\xi_{h}\right)$ for all $\xi_{h} \in W_{h}$, where $\psi_{h}$ is defined by

$$
\frac{1}{\Delta t} A^{h}\left(\psi_{h}^{n}, \phi_{h}\right)+\nu B^{h}\left(\psi_{h}^{n}, \phi_{h}\right)+C^{h}\left(\xi_{h} ; \psi_{h}^{n}, \phi_{h}\right)=F^{h}\left(\phi_{h}\right)+\frac{1}{\Delta t} A^{h}\left(\psi_{h}^{n-1}, \phi_{h}\right) .
$$

The proof of the result will be divided in three steps. We first define a mapping $\mathcal{F}$ from $W_{h}$ to $W_{h}$ and prove that the mapping is well-defined and maps a ball $\mathcal{B}_{\mathcal{R}}$ to a ball $\mathcal{B}_{\mathcal{R}}$. In second stage, we prove that the mapping is continuous. Then, from Brouwer's Theorem, we deduce that $\mathcal{F}$ has a fixed point inside the ball $\mathcal{B}_{\mathcal{R}}$ which is the solution of the fully-discrete scheme (4.7). Finally, using assumption (4.8), we prove that the solution is unique.

Well possedness of $\mathcal{F}$ : Since the bilinear form $B^{h}\left(\phi_{h}, \phi_{h}\right)$ is elliptic (cf. Lem. 3.7), (3.18) and the fact that $A^{h}\left(\phi_{h}, \phi_{h}\right)>0$, we have that problem (4.9) is well-posed, which follows from the Lax-Milgram Theorem. Further, to present the analysis we denote by $\mathcal{D}_{t} \psi_{h}^{n}:=\frac{\psi_{h}^{n}-\psi_{h}^{n-1}}{\Delta t}$.

Now, we will construct a ball with radius $\mathcal{R}$ say $\mathcal{B}_{\mathcal{R}}$ such that $\mathcal{F}: \mathcal{B}_{\mathcal{R}} \rightarrow \mathcal{B}_{\mathcal{R}}$. We consider $\phi_{h}=\mathcal{D}_{t} \psi_{h}^{n}$ in (4.9) and obtain

$$
A^{h}\left(\mathcal{D}_{t} \psi_{h}^{n}, \mathcal{D}_{t} \psi_{h}^{n}\right)+\nu B^{h}\left(\psi_{h}^{n}, \mathcal{D}_{t} \psi_{h}^{n}\right)+C^{h}\left(\xi_{h} ; \psi_{h}^{n}, \mathcal{D}_{t} \psi_{h}^{n}\right)=F^{h}\left(\mathcal{D}_{t} \psi_{h}^{n}\right)
$$

An application of the stability property of the discrete bilinear forms (3.12), (3.13), and (3.18) yields

$$
\alpha_{1}\left\|\operatorname{curl} \mathcal{D}_{t} \psi_{h}^{n}\right\|_{0, \Omega}^{2}+\frac{\alpha_{3} \nu}{\Delta t}\left\|\psi_{h}^{n}\right\|_{2, \Omega}^{2} \leq\left|F^{h}\left(\mathcal{D}_{t} \psi_{h}^{n}\right)\right|+\frac{\nu}{\Delta t} B^{h}\left(\psi_{h}^{n}, \psi_{h}^{n-1}\right)+\frac{1}{\Delta t} C^{h}\left(\xi_{h} ; \psi_{h}^{n}, \psi_{h}^{n-1}\right) .
$$

By exploiting Cauchy-Schwarz inequality and boundedness of the $L^{2}$-projection operator $\boldsymbol{\Pi}_{K}^{k-1}$, we obtain

$$
\left|F^{h}\left(\mathcal{D}_{t} \psi_{h}^{n}\right)\right|=\left|\sum_{K \in \mathcal{T}_{h}} \int_{K} \boldsymbol{\Pi}_{K}^{k-1} \mathbf{f} \cdot \operatorname{curl} \mathcal{D}_{t} \psi_{h}^{n}\right| \leq\|\mathbf{f}\|_{0, \Omega}\left\|\operatorname{curl} \mathcal{D}_{t} \psi_{h}^{n}\right\|_{0, \Omega} .
$$

Using the continuity property of $B^{h}(\cdot, \cdot)(c f .(3.14))$, we get

$$
\left|B^{h}\left(\psi_{h}^{n}, \psi_{h}^{n-1}\right)\right| \leq C_{B}\left\|\psi_{h}^{n}\right\|_{2, \Omega}\left\|\psi_{h}^{n-1}\right\|_{2, \Omega}
$$


Inserting (4.11) and (4.12) in (4.10), and using boundedness of the trilinear term $C^{h}(\cdot ; \cdot, \cdot)(c f$. Lem. 3.8), we obtain

$$
\begin{aligned}
\alpha_{1}\left\|\operatorname{curl} \mathcal{D}_{t} \psi_{h}^{n}\right\|_{0, \Omega}^{2}+\frac{\alpha_{3} \nu}{\Delta t}\left\|\psi_{h}^{n}\right\|_{2, \Omega}^{2} \leq & \left(\|\mathbf{f}\|_{0, \Omega}\left\|\operatorname{curl} \mathcal{D}_{t} \psi_{h}^{n}\right\|_{0, \Omega}+\frac{C_{B} \nu}{\Delta t}\left\|\psi_{h}^{n}\right\|_{2, \Omega}\left\|\psi_{h}^{n-1}\right\|_{2, \Omega}\right. \\
& \left.+\frac{\hat{C}}{\Delta t}\left\|\xi_{h}\right\|_{2, \Omega}\left\|\psi_{h}^{n}\right\|_{2, \Omega}\left\|\psi_{h}^{n-1}\right\|_{2, \Omega}\right)
\end{aligned}
$$

Upon employing Young's inequality, we obtain

$$
\begin{aligned}
\frac{\alpha_{1}}{2}\left\|\operatorname{curl} \mathcal{D}_{t} \psi_{h}^{n}\right\|_{0, \Omega}^{2}+\frac{\alpha_{3} \nu}{\Delta t}\left\|\psi_{h}^{n}\right\|_{2, \Omega}^{2} \leq & \frac{1}{2 \alpha_{1}}\|\mathbf{f}\|_{0, \Omega}^{2}+\frac{C_{B} \nu}{\Delta t}\left\|\psi_{h}^{n}\right\|_{2, \Omega}\left\|\psi_{h}^{n-1}\right\|_{2, \Omega} \\
& +\frac{\hat{C}}{\Delta t}\left\|\xi_{h}\right\|_{2, \Omega}\left\|\psi_{h}^{n}\right\|_{2, \Omega}\left\|\psi_{h}^{n-1}\right\|_{2, \Omega} .
\end{aligned}
$$

Since the term $\frac{\alpha_{1}}{2}\left\|\operatorname{curl} \mathcal{D}_{t} \psi_{h}^{n}\right\|_{0, \Omega}^{2}$ is positive, we can neglect the term and obtain

$$
\alpha_{3} \nu\left\|\psi_{h}^{n}\right\|_{2, \Omega}^{2} \leq \frac{\Delta t}{2 \alpha_{1}}\|\mathbf{f}\|_{0, \Omega}^{2}+C_{B} \nu\left\|\psi_{h}^{n}\right\|_{2, \Omega}\left\|\psi_{h}^{n-1}\right\|_{2, \Omega}+\hat{C}\left\|\xi_{h}\right\|_{2, \Omega}\left\|\psi_{h}^{n}\right\|_{2, \Omega}\left\|\psi_{h}^{n-1}\right\|_{2, \Omega}
$$

Exploiting Young's inequality and kick-back argument, we obtain

$$
\begin{aligned}
\frac{\alpha_{3} \nu}{2}\left\|\psi_{h}^{n}\right\|_{2, \Omega}^{2} & \leq \frac{\Delta t}{2 \alpha_{1}}\|\mathbf{f}\|_{0, \Omega}^{2}+\frac{C_{B}^{2} \nu}{\alpha_{3}}\left\|\psi_{h}^{n-1}\right\|_{2, \Omega}^{2}+\frac{\hat{C}^{2}}{\alpha_{3} \nu}\left\|\xi_{h}\right\|_{2, \Omega}^{2}\left\|\psi_{h}^{n-1}\right\|_{2, \Omega}^{2} \\
& \leq \frac{\Delta t}{2 \alpha_{1}}\|\mathbf{f}\|_{L^{\infty}\left(0, t_{n-1} ; L^{2}(\Omega)\right)}^{2}+\left(\frac{C_{B}^{2} \nu}{\alpha_{3}}+\frac{\hat{C}^{2}}{\alpha_{3} \nu}\left\|\xi_{h}\right\|_{2, \Omega}^{2}\right)\left\|\psi_{h}\right\|_{L^{\infty}\left(0, t_{n-1} ; H^{2}(\Omega)\right)}^{2} .
\end{aligned}
$$

Moreover, we adopt that $\left\|\xi_{h}\right\|_{2, \Omega} \leq \mathcal{R}$. Then from (4.14), we derive as

$$
\frac{\Delta t}{\alpha_{1} \alpha_{3} \nu}\|\mathbf{f}\|_{L^{\infty}\left(0, t_{n-1} ; L^{2}(\Omega)\right)}^{2}+\left(\frac{2 C_{B}^{2}}{\alpha_{3}^{2}}+\frac{2 \hat{C}^{2}}{\alpha_{3}^{2} \nu^{2}} \mathcal{R}^{2}\right)\left\|\psi_{h}\right\|_{L^{\infty}\left(0, t_{n-1} ; H^{2}(\Omega)\right)}^{2} \leq \mathcal{R}^{2} .
$$

Upon writing explicitly (4.15), we obtain

$$
\mathcal{R}^{2}=\frac{\left(\frac{\Delta t}{\alpha_{1} \alpha_{3} \nu}\|\mathbf{f}\|_{L^{\infty}\left(0, t_{n-1} ; L^{2}(\Omega)\right)}^{2}+\frac{2 C_{B}^{2}}{\alpha_{3}^{2}}\left\|\psi_{h}\right\|_{L^{\infty}\left(0, t_{n-1} ; H^{2}(\Omega)\right)}^{2}\right)}{\left(1-\frac{2 \hat{C}^{2}}{\alpha_{3}^{2} \nu^{2}}\left\|\psi_{h}\right\|_{L^{\infty}\left(0, t_{n-1} ; H^{2}(\Omega)\right)}^{2}\right)} .
$$

Now, according to assumption (4.8), the term $\left(1-\frac{2 \hat{C}^{2}}{\alpha_{3}^{2} \nu^{2}}\left\|\psi_{h}\right\|_{L^{\infty}\left(0, t_{n-1} ; H^{2}(\Omega)\right)}^{2}\right)$ is positive and consequently, we define $\mathcal{B}_{\mathcal{R}}:=\left\{v_{h} \in W_{h}:\left\|v_{h}\right\|_{2, \Omega} \leq \mathcal{R}\right\}$. Therefore, we deduce that $\mathcal{F}: \mathcal{B}_{\mathcal{R}} \rightarrow \mathcal{B}_{\mathcal{R}}$ is well defined.

Continuity of $\mathcal{F}$ : Let $\varepsilon>0$ be a small number and let $\xi_{h}^{\star}, \xi_{h}$ be two elements in $\mathcal{B}_{\mathcal{R}}$ such that $\psi_{h}^{\star}:=\mathcal{F}\left(\xi_{h}^{\star}\right)$ and $\psi_{h}:=\mathcal{F}\left(\xi_{h}\right)$ and $\left\|\Delta\left(\xi_{h}^{\star}-\xi_{h}\right)\right\|_{0, \Omega}<\delta$. Then, from (4.9), we have

$$
A^{h}\left(\psi_{h}-\psi_{h}^{\star}, \phi_{h}\right)+\Delta t \nu B^{h}\left(\psi_{h}-\psi_{h}^{\star}, \phi_{h}\right)+\Delta t\left(C^{h}\left(\xi_{h} ; \psi_{h}, \phi_{h}\right)-C^{h}\left(\xi_{h}^{\star} ; \psi_{h}^{\star}, \phi_{h}\right)\right)=0 .
$$

Putting $\phi_{h}=\left(\psi_{h}-\psi_{h}^{\star}\right) \in W_{h}$ in (4.16), we obtain

$$
A^{h}\left(\psi_{h}-\psi_{h}^{\star}, \psi_{h}-\psi_{h}^{\star}\right)+\Delta t \nu B^{h}\left(\psi_{h}-\psi_{h}^{\star}, \psi_{h}-\psi_{h}^{\star}\right)+\Delta t\left(C^{h}\left(\xi_{h} ; \psi_{h}, \psi_{h}-\psi_{h}^{\star}\right)-C^{h}\left(\xi_{h}^{\star} ; \psi_{h}^{\star}, \psi_{h}-\psi_{h}^{\star}\right)\right)=0 .
$$


An application of stability property of $A^{h}(\cdot, \cdot)$ and $B^{h}(\cdot, \cdot)$ yields

$$
\alpha_{1}\left\|\operatorname{curl}\left(\psi_{h}-\psi_{h}^{\star}\right)\right\|_{0, \Omega}^{2}+\Delta t \nu \alpha_{3}\left\|\Delta\left(\psi_{h}-\psi_{h}^{\star}\right)\right\|_{0, \Omega}^{2} \leq \Delta t\left|C^{h}\left(\xi_{h}^{\star} ; \psi_{h}^{\star}, \psi_{h}-\psi_{h}^{\star}\right)-C^{h}\left(\xi_{h} ; \psi_{h}, \psi_{h}-\psi_{h}^{\star}\right)\right| .
$$

The difference on the right hand side above can be bound as follows:

$$
\begin{aligned}
& \left|\sum_{K \in \mathcal{T}_{h}}\left(\int_{K} \Delta \Pi_{K}^{k, \Delta} \xi_{h} \Pi_{K}^{k-1} \operatorname{curl} \psi_{h} \cdot \Pi_{K}^{k-1} \nabla\left(\psi_{h}-\psi_{h}^{\star}\right)-\int_{K} \Delta \Pi_{K}^{k, \Delta} \xi_{h}^{\star} \Pi_{K}^{k-1} \operatorname{curl} \psi_{h}^{\star} \cdot \Pi_{K}^{k-1} \nabla\left(\psi_{h}-\psi_{h}^{\star}\right)\right)\right| \\
& \leq \sum_{K \in \mathcal{T}_{h}} \underbrace{\left|\left(\int_{K} \Delta \Pi_{K}^{k, \Delta} \xi_{h} \Pi_{K}^{k-1} \operatorname{curl} \psi_{h} \cdot \Pi_{K}^{k-1} \nabla\left(\psi_{h}-\psi_{h}^{\star}\right)-\int_{K} \Delta \Pi_{K}^{k, \Delta} \xi_{h}^{\star} \Pi_{K}^{k-1} \operatorname{curl} \psi_{h} \cdot \Pi_{K}^{k-1} \nabla\left(\psi_{h}-\psi_{h}^{\star}\right)\right)\right|}_{=: T_{1}} \\
& +\underbrace{\left|\left(\int_{K} \Delta \Pi_{K}^{k, \Delta} \xi_{h}^{\star} \Pi_{K}^{k-1} \operatorname{curl} \psi_{h} \cdot \Pi_{K}^{k-1} \nabla\left(\psi_{h}-\psi_{h}^{\star}\right)-\int_{K} \Delta \Pi_{K}^{k, \Delta} \xi_{h}^{\star} \Pi_{K}^{k-1} \operatorname{curl} \psi_{h}^{\star} \cdot \Pi_{K}^{k-1} \nabla\left(\psi_{h}-\psi_{h}^{\star}\right)\right)\right|}_{=: T_{2}} .
\end{aligned}
$$

Using Hölder's inequality and the boundedness of the projection operator $\Pi_{K}^{k, \Delta}$, the term $T_{1}$ can be bounded as follows:

$$
\begin{aligned}
\left|T_{1}\right| & \leq\left\|\Delta \Pi_{K}^{k, \Delta} \xi_{h}-\Delta \Pi_{K}^{k, \Delta} \xi_{h}^{\star}\right\|_{0, K}\left\|\Pi_{K}^{k-1} \operatorname{curl} \psi_{h}\right\|_{L^{4}(K)}\left\|\Pi_{K}^{k-1} \nabla\left(\psi_{h}-\psi_{h}^{\star}\right)\right\|_{L^{4}(K)} \\
& \leq\left\|\Delta\left(\xi_{h}-\xi_{h}^{\star}\right)\right\|_{0, K}\left\|\operatorname{curl} \psi_{h}\right\|_{L^{4}(K)}\left\|\nabla\left(\psi_{h}-\psi_{h}^{\star}\right)\right\|_{L^{4}(K)}
\end{aligned}
$$

Suming the above inequality for all elements $K$ and using the Sobolev's embedding theorem,

$$
\sum_{K \in \mathcal{T}_{h}}\left|T_{1}\right| \leq \widehat{C}\left\|\Delta\left(\xi_{h}-\xi_{h}^{\star}\right)\right\|_{0, \Omega}\left\|\operatorname{curl} \psi_{h}\right\|_{1, \Omega}\left\|\nabla\left(\psi_{h}-\psi_{h}^{\star}\right)\right\|_{1, \Omega}
$$

Now, using (3.18), we deduce that the term $T_{2}=0$.

Inserting (4.18) into (4.17), we obtain

$$
\alpha_{1}\left\|\operatorname{curl}\left(\psi_{h}-\psi_{h}^{\star}\right)\right\|_{0, \Omega}^{2}+\Delta t \alpha_{3} \nu\left\|\psi_{h}-\psi_{h}^{\star}\right\|_{2, \Omega}^{2} \leq \widehat{C} \Delta t \delta\left\|\operatorname{curl} \psi_{h}\right\|_{1, \Omega}\left\|\nabla\left(\psi_{h}-\psi_{h}^{\star}\right)\right\|_{1, \Omega} .
$$

Since the term $\alpha_{1}\left\|\operatorname{curl}\left(\psi_{h}-\psi_{h}^{\star}\right)\right\|_{0, \Omega}^{2}$ is positive, we deduce that

$$
\left\|\psi_{h}-\psi_{h}^{\star}\right\|_{2, \Omega} \leq \frac{\widehat{C} \delta}{\alpha_{3} \nu}\left\|\operatorname{curl} \psi_{h}\right\|_{1, \Omega} .
$$

Hence, using that $\psi_{h} \in \mathcal{B}_{\mathcal{R}}$, we conclude that $\left\|\psi_{h}-\psi_{h}^{\star}\right\|_{2, \Omega}<\varepsilon$ if

$$
\delta<\frac{\alpha_{3} \nu \varepsilon}{\widehat{C} \mathcal{R}}
$$

which implies that the function $\mathcal{F}$ is continuous.

Therefore, we claim that the function $\mathcal{F}$ has a fixed point $\psi_{h} \in \mathcal{B}_{\mathcal{R}}$ from Brouwer's fixed point theorem (cf. Thm. 4.1) such that $\psi_{h}=\mathcal{F}\left(\psi_{h}\right)$. Hence the fully-discrete scheme (4.7) has a solution. Now we proceed to show that the solution is unique.

Uniqueness of solution: Let $\psi_{h}^{1}, \psi_{h}^{2} \in \mathcal{B}_{\mathcal{R}}$ be two solutions of (4.7). Then from (4.9), we have

$$
A^{h}\left(\psi_{h}^{1}-\psi_{h}^{2}, \phi_{h}\right)+\Delta t \nu B^{h}\left(\psi_{h}^{1}-\psi_{h}^{2}, \phi_{h}\right)+\Delta t\left(C^{h}\left(\psi_{h}^{1} ; \psi_{h}^{1}, \phi_{h}\right)-C^{h}\left(\psi_{h}^{2} ; \psi_{h}^{2}, \phi_{h}\right)\right)=0 .
$$


To bound the nonlinear term in (4.19), we add and substract $C^{h}\left(\psi_{h}^{2} ; \psi_{h}^{1}, \phi_{h}\right)$ in the above equality, to get

$$
\begin{aligned}
C^{h}\left(\psi_{h}^{1} ; \psi_{h}^{1}, \phi_{h}\right)-C^{h}\left(\psi_{h}^{2} ; \psi_{h}^{2}, \phi_{h}\right)= & C^{h}\left(\psi_{h}^{1} ; \psi_{h}^{1}, \phi_{h}\right)-C^{h}\left(\psi_{h}^{2} ; \psi_{h}^{1}, \phi_{h}\right) \\
& +C^{h}\left(\psi_{h}^{2} ; \psi_{h}^{1}, \phi_{h}\right)-C^{h}\left(\psi_{h}^{2} ; \psi_{h}^{2}, \phi_{h}\right) .
\end{aligned}
$$

Using Hölder's inequality and Sobolev embedding theorem, the first two terms in (4.20) can be bounded as follows

$$
\begin{aligned}
\left|C^{h}\left(\psi_{h}^{1} ; \psi_{h}^{1}, \phi_{h}\right)-C^{h}\left(\psi_{h}^{2} ; \psi_{h}^{1}, \phi_{h}\right)\right| & =\left|\sum_{K \in \mathcal{T}_{h}} \int_{K}\left(\Delta \Pi_{K}^{k, \Delta}\left(\psi_{h}^{1}-\psi_{h}^{2}\right) \boldsymbol{\Pi}_{K}^{k-1} \operatorname{curl} \psi_{h}^{1} \cdot \Pi_{K}^{k-1} \nabla \phi_{h}\right)\right| \\
& \leq \widehat{C}\left\|\psi_{h}^{1}-\psi_{h}^{2}\right\|_{2, \Omega}\left\|\operatorname{curl} \psi_{h}^{1}\right\|_{1, \Omega}\left\|\nabla \phi_{h}\right\|_{1, \Omega} .
\end{aligned}
$$

The last two terms on the right hand side of (4.20) can be written as follows,

$$
C^{h}\left(\psi_{h}^{2} ; \psi_{h}^{1}, \phi_{h}\right)-C^{h}\left(\psi_{h}^{2} ; \psi_{h}^{2}, \phi_{h}\right)=C^{h}\left(\psi_{h}^{2} ; \psi_{h}^{1}-\psi_{h}^{2}, \phi_{h}\right)
$$

Taking $\phi_{h}=\left(\psi_{h}^{1}-\psi_{h}^{2}\right) \in W_{h}$ in (4.19), we have that the right hand side above vanish (cf. (3.18)), and using the stability property of the discrete bilinear forms $A^{h}(\cdot, \cdot)$ and $B^{h}(\cdot, \cdot)$, and Young's inequality, we obtain

$$
\alpha_{1}\left\|\operatorname{curl}\left(\psi_{h}^{1}-\psi_{h}^{2}\right)\right\|_{0, \Omega}^{2}+\alpha_{3} \Delta t \nu\left\|\psi_{h}^{1}-\psi_{h}^{2}\right\|_{2, \Omega}^{2} \leq \Delta t \widehat{C}\left\|\psi_{h}^{1}-\psi_{h}^{2}\right\|_{2, \Omega}\left\|\operatorname{curl} \psi_{h}^{1}\right\|_{1, \Omega}\left\|\nabla\left(\psi_{h}^{1}-\psi_{h}^{2}\right)\right\|_{1, \Omega} .
$$

An application of kick back arguments, we obtain

$$
\alpha_{1}\left\|\operatorname{curl}\left(\psi_{h}^{1}-\psi_{h}^{2}\right)\right\|_{0, \Omega}^{2}+\Delta t\left(\alpha_{3} \nu-\widehat{C} \mathcal{R}\right)\left\|\psi_{h}^{1}-\psi_{h}^{2}\right\|_{2, \Omega}^{2} \leq 0
$$

Now, according to assumption (4.8), and for sufficiently small values of $\Delta t,\left(\alpha_{3} \nu-\widehat{C} \mathcal{R}\right)>0$. Hence, we have $\left\|\psi_{h}^{1}-\psi_{h}^{2}\right\|_{2, \Omega}=0$; therefore $\psi_{h}^{1}=\psi_{h}^{2}$, and we conclude the proof.

Remark 4.3. In Theorem 4.2, we have proved the fully-discrete scheme (4.7) has unique solution based on certain feasible assumption on the viscosity $\nu(c f$. (4.8)) and for sufficiently small values of time-step $\Delta t$. In particular, for sufficiently large values of $\nu$, we have

$$
\left(\alpha_{3}^{2} \nu^{2}-\frac{2 \hat{C}^{2} C_{B}^{2}}{\alpha_{3}^{2}}\left\|\psi_{h}\right\|_{L^{\infty}\left(0, t_{n-1} ; H^{2}(\Omega)\right)}^{2}\right)>0 .
$$

Then for sufficiently small values of $\Delta t$, we have

$$
\nu\left(\alpha_{3}^{2} \nu^{2}-\frac{2 \hat{C}^{2} C_{B}^{2}}{\alpha_{3}^{2}}\left\|\psi_{h}\right\|_{L^{\infty}\left(0, t_{n-1} ; H^{2}(\Omega)\right)}^{2}\right)>\frac{\hat{C}^{2}}{\alpha_{1} \alpha_{3}} \Delta t\|\mathbf{f}\|_{L^{\infty}\left(0, t_{n-1} ; L^{2}(\Omega)\right)}^{2},
$$

which implies

$$
\left(\alpha_{3} \nu-\widehat{C} \mathcal{R}\right)>0
$$

\section{Convergence analysis}

In this section, we will derive a priori error estimation for the virtual element semi-discrete and fully-discrete schemes. With this aim, first we introduce a discrete energy projection operator $\mathcal{S}_{h}: H_{0}^{2}(\Omega) \rightarrow W_{h}$, which is defined as follows:

$$
B^{h}\left(\mathcal{S}_{h} u, w_{h}\right)=B\left(u, w_{h}\right) \quad \forall w_{h} \in W_{h}
$$


Upon exploiting the energy projection operator $\mathcal{S}_{h}$, we will split the error of the stream-function as

$$
\psi-\psi_{h}=\psi-\mathcal{S}_{h} \psi+\mathcal{S}_{h} \psi-\psi_{h}
$$

Next, we define

$$
\begin{aligned}
\rho_{h} & :=\psi-\mathcal{S}_{h} \psi, \\
\theta_{h} & :=\psi_{h}-\mathcal{S}_{h} \psi .
\end{aligned}
$$

In what follows, we will prove approximations properties for $\mathcal{S}_{h}$, thus the first term $\rho_{h}$ will be easily bounded. Then, using the continuous problem (2.3) and the polynomial approximation properties, we bound the term $\theta_{h}$. In this regard, we introduce the polynomial approximation property and the interpolation operator $\psi_{I}$ on the virtual element space $W_{h}$. Further, to derive the a priori error estimations of the semi-discrete and fully-discrete schemes, some additional results are needed which will be presented in the next subsection.

\subsection{Preliminary results}

We start with the following approximation result, on star-shaped polygons, which is derived by interpolation between Sobolev spaces (see for instance [28], Thm. I.1.4 from the analogous result for integer values of $s$ ). We mention that this result has been stated in Proposition 4.2 of [6] for integer values and follows from the classical Scott-Dupont theory (see [15] and [5], Prop. 3.1):

Proposition 5.1. There exists a constant $C>0$, independent of mesh size $h$ but depends on mesh regularity parameter $C_{\mathcal{T}}$ (Assumption 3.1) such that for every $v \in H^{\delta}(K)$ there exists $v_{\pi} \in \mathcal{P}_{k}(K), k \geq 0$ such that

$$
\left\|v-v_{\pi}\right\|_{\ell, K} \leq C h_{K}^{\delta-\ell}\|v\|_{\delta, K} \quad 0 \leq \delta \leq k+1, \ell=0, \ldots,[\delta],
$$

with $[\delta]$ denoting largest integer equal or smaller than $\delta \in \mathbb{R}$.

Now, we present an interpolation result in the virtual space $W_{h}$ (see $\left.[5,10]\right)$.

Proposition 5.2. Assume $\boldsymbol{A} 1$ and $\boldsymbol{A} 2$ are satisfied, then for all $v \in H^{\delta}(K)$ there exist $v_{I} \in W_{h}$ and $C>0$ independent of $h$ such that

$$
\left\|v-v_{I}\right\|_{\ell, K} \leq C h_{K}^{\delta-\ell}\|v\|_{\delta, K}, \quad \ell=0,1,2, \quad 2 \leq \delta \leq k+1,
$$

where $C$ is independent of mesh size $h$ but depends on mesh regularity parameter $C_{\mathcal{T}}$ (Assumption 3.1).

Next, in order to prove the convergence of our method, we introduce the following broken $H^{\ell}$-seminorm $(\ell=1,2)$ :

$$
|v|_{\ell, h}:=\left(\sum_{K \in \mathcal{T}_{h}}|v|_{\ell, K}^{2}\right)^{1 / 2},
$$

which is well defined for every $v \in L^{2}(\Omega)$ such that $\left.v\right|_{K} \in H^{\ell}(K)$ for all polygon $K \in \mathcal{T}_{h}$.

In order to obtain the error estimates, we prove the following approximation properties of the discrete projection operator $\mathcal{S}_{h}(c f .(5.1))$.

Lemma 5.3. For each $u \in H_{0}^{2}(\Omega) \cap H^{2+s}(\Omega)$, with $1 / 2<s \leq k-1$, there exists a unique function $\mathcal{S}_{h}(u) \in W_{h}$, such that the following approximation properties hold:

(1) There exists a positive constant $C$, independent of $h$, such that

$$
\left\|u-\mathcal{S}_{h}(u)\right\|_{2, \Omega} \leq C h^{s}\|u\|_{2+s, \Omega}
$$


(2) There exist a positive constant $C$ and $\tilde{s} \in(1 / 2,1]$, independent of $h$, such that

$$
\left\|u-\mathcal{S}_{h}(u)\right\|_{1, \Omega} \leq C h^{\tilde{s}+s}\|u\|_{2+s, \Omega} .
$$

Proof. We begin by proving (1). Since the bilinear form $B^{h}(\cdot, \cdot)$ is bounded and coercive in $W_{h}$ and the function $B(u, \cdot)$ is continuous on $W_{h}$ hence there exists an unique solution of the variational formulation (5.1).

Now, we proceed to prove the approximation results. In this direction, we rewrite the term $u-\mathcal{S}_{h}(u)$ as follows.

$$
u-\mathcal{S}_{h}(u)=u-u_{I}+u_{I}-\mathcal{S}_{h}(u),
$$

where $u_{I} \in W_{h}$ is the interpolation operator introduced in Proposition 5.2.

The estimation of $u-u_{I}$ is known, hence we proceed to bound the term $\xi_{h}:=\left(\mathcal{S}_{h}(u)-u_{I}\right) \in W_{h}$. Using coercivity (cf. Lem. 3.7), we have

$$
\begin{aligned}
\alpha\left|\xi_{h}\right|_{2, \Omega}^{2} & \leq B^{h}\left(\xi_{h}, \xi_{h}\right) \\
& =B^{h}\left(\mathcal{S}_{h}(u), \xi_{h}\right)-B^{h}\left(u_{I}, \xi_{h}\right) \\
& =B\left(u, \xi_{h}\right)-\sum_{K \in \mathcal{T}_{h}}\left(B_{K}^{h}\left(u_{I}-u_{\pi}, \xi_{h}\right)+B_{K}^{h}\left(u_{\pi}, \xi_{h}\right)\right) \\
& =B\left(u, \xi_{h}\right)-\sum_{K \in \mathcal{T}_{h}}\left(B_{K}^{h}\left(u_{I}-u_{\pi}, \xi_{h}\right)+B_{K}\left(u_{\pi}, \xi_{h}\right)\right) \\
& =\sum_{K \in \mathcal{T}_{h}}\left(B_{K}^{h}\left(u_{\pi}-u_{I}, \xi_{h}\right)+B_{K}\left(u-u_{\pi}, \xi_{h}\right)\right),
\end{aligned}
$$

where we have added and subtracted $u_{\pi} \in \mathcal{P}_{k}(K), k \geq 2$ and then we have used (3.11). Using the continuity property of the bilinear form $B^{h}(\cdot, \cdot)$ and Cauchy-Schwarz inequality, we have

$$
\alpha\left|\xi_{h}\right|_{2, \Omega}^{2} \leq C\left(\left|u_{\pi}-u\right|_{2, h}+\left|u-u_{I}\right|_{2, \Omega}\right)\left|\xi_{h}\right|_{2, \Omega} .
$$

Now, an application of the approximation properties of the interpolation operator and the projection operator $u_{I}$ and $u_{\pi}$, respectively, we obtain

$$
\alpha\left|\xi_{h}\right|_{2, \Omega} \leq C h^{s}\|u\|_{2+s, \Omega} .
$$

Thus, we have that

$$
\left|u-\mathcal{S}_{h}(u)\right|_{2, \Omega} \leq C h^{s}\|u\|_{2+s, \Omega} .
$$

Now, we proceed to prove (2). Let $\phi \in H_{0}^{2}(\Omega)$ be the solution of the auxiliary variational problem: find $\phi$ such that

$$
B(\phi, v)=\int_{\Omega} \nabla\left(u-\mathcal{S}_{h}(u)\right) \cdot \nabla v \quad \forall v \in H_{0}^{2}(\Omega),
$$

where $B(\cdot, \cdot)$ is the bilinear form defined in $(2.6)$.

As a consequence of a classical regularity result for the biharmonic problem with its right hand side in $H^{-1}(\Omega):=\left[H_{0}^{1}(\Omega)\right]^{\prime}(c f .[29])$, there exists $\tilde{s} \in(1 / 2,1]$ such that $\phi \in H^{2+\tilde{s}}(\Omega)$ and

$$
\|\phi\|_{2+\tilde{s}, \Omega} \leq C\left|u-\mathcal{S}_{h}(u)\right|_{1, \Omega} .
$$

Now, let $\phi_{I} \in W_{h}$ be such that Proposition 5.2 holds true. Taking $v:=\left(u-\mathcal{S}_{h}(u)\right) \in H_{0}^{2}(\Omega)$ as test function in (5.7), using the symmetry of the bilinear form and adding and subtracting $\phi_{I}$, we obtain

$$
\begin{aligned}
\left|u-\mathcal{S}_{h}(u)\right|_{1, \Omega}^{2} & \leq B\left(u-\mathcal{S}_{h}(u), \phi\right) \\
& =B\left(u-\mathcal{S}_{h}(u), \phi-\phi_{I}\right)+B\left(u-\mathcal{S}_{h}(u), \phi_{I}\right) \\
& =B\left(u-\mathcal{S}_{h}(u), \phi-\phi_{I}\right)+B\left(u, \phi_{I}\right)-B\left(\mathcal{S}_{h}(u), \phi_{I}\right) \\
& =B\left(u-\mathcal{S}_{h}(u), \phi-\phi_{I}\right)+B^{h}\left(\mathcal{S}_{h}(u), \phi_{I}\right)-B\left(\mathcal{S}_{h}(u), \phi_{I}\right) \\
& =: T_{1}+T_{2},
\end{aligned}
$$


where we have used the definition of the continuous (see (2.5)) and discrete problems (see (5.1)).

Now, we bound the terms $T_{1}$ and $T_{2}$ separately. We start with $T_{1}$ as follows.

$$
\begin{aligned}
T_{1}=B\left(u-\mathcal{S}_{h}(u), \phi-\phi_{I}\right) & \leq\left\|u-\mathcal{S}_{h}(u)\right\|_{2, \Omega}\left\|\phi-\phi_{I}\right\|_{2, \Omega} \\
& \leq C h^{s}\left(\|u\|_{2+s, \Omega}\right) h^{\tilde{s}}\|\phi\|_{2+\tilde{s}, \Omega} \\
& \leq C h^{\tilde{s}+s}\left(\|u\|_{2+s, \Omega}\right)\left|u-\mathcal{S}_{h}(u)\right|_{1, \Omega},
\end{aligned}
$$

where we have used the continuity of bilinear form $B(\cdot, \cdot),(5.6)$ and Proposition 5.2.

Now, we continue with the term $T_{2}$ in (5.9). Let $u_{\pi} \in \mathcal{P}_{k}(K), \phi_{\pi} \in \mathcal{P}_{2}(K)$ such that Proposition 5.1 holds true with respect to $u$ and $\phi$, respectively. Using (3.11), we have

$$
\begin{aligned}
T_{2} & =\sum_{K \in \mathcal{T}_{h}}\left[B_{K}^{h}\left(\mathcal{S}_{h}(u)-u_{\pi}, \phi_{I}-\phi_{\pi}\right)+B_{K}\left(u_{\pi}-\mathcal{S}_{h}(u), \phi_{I}-\phi_{\pi}\right)\right] \\
& \leq \sum_{K \in \mathcal{T}_{h}} C\left\|\mathcal{S}_{h}(u)-u_{\pi}\right\|_{2, K}\left\|\phi_{I}-\phi_{\pi}\right\|_{2, K} \\
& \leq \sum_{K \in \mathcal{T}_{h}} C\left(\left\|\mathcal{S}_{h}(u)-u\right\|_{2, K}+\left\|u-u_{\pi}\right\|_{2, K}\right)\left(\left\|\phi_{I}-\phi\right\|_{2, K}+\left\|\phi-\phi_{\pi}\right\|_{2, K}\right) \\
& \leq \sum_{K \in \mathcal{T}_{h}} C\left(\left\|\mathcal{S}_{h}(u)-u\right\|_{2, K}+h_{K}^{s}\|u\|_{2+s, K}\right)\left(\left\|\phi_{I}-\phi\right\|_{2, K}+C h_{K}^{\tilde{s}}\|\phi\|_{2+\tilde{s}, K}\right) \\
& \leq C\left(\left\|\mathcal{S}_{h}(u)-u\right\|_{2, \Omega}+C h^{s}\|u\|_{2+s, \Omega}\right)\left(C h^{\tilde{s}}\|\phi\|_{2+\tilde{s}, \Omega}+\left\|\phi_{I}-\phi\right\|_{2, \Omega}\right) \\
& \leq C\left(h^{s}\|u\|_{2+s, \Omega}\right) C h^{\tilde{s}}\|\phi\|_{2+\tilde{s}, \Omega} \\
& \leq C h^{\tilde{s}+s}\left(\|u\|_{2+s, \Omega}\right)\left|u-\mathcal{S}_{h}(u)\right|_{1, \Omega},
\end{aligned}
$$

where we have used continuity of local bilinear forms $B_{K}(\cdot, \cdot)$ and $B_{K}^{h}(\cdot, \cdot)$, Proposition 5.1, (5.6) and (5.8). Thus, equation (5.3) follows from (5.9) to (5.11). The proof is complete.

\subsection{Error estimation for semi-discrete scheme}

In this section, we will derive the error estimation for the semi-discrete scheme (cf. (4.1)). With this end, we state the following lemma.

Lemma 5.4. Let $\psi(t) \in W$ be the solution of problem (2.5). Assume that $\psi(t) \in H^{2+r}(\Omega)$, for $\frac{1}{2}<r \leq k-1$, for almost all $t \in[0, T]$. Then, there exists a positive generic constant $C$, which could be depended on mesh regularity Assumption 3.1, Sobolev regularity of the solution $\psi$, but independent of mesh size $h$ such that

$$
\left|C\left(\psi ; \psi, \phi_{h}\right)-C^{h}\left(\psi ; \psi, \phi_{h}\right)\right| \leq C h^{r}\left(|\psi|_{1+r, \Omega}+\|\psi\|_{2, \Omega}\right)|\psi|_{2+r, \Omega}\left\|\phi_{h}\right\|_{2, \Omega} \quad \forall \phi_{h} \in W_{h} .
$$

Proof. Using the definition of the continuous nonlinear term $C(\cdot ; \cdot, \cdot)$ (see $(2.4))$ and discrete nonlinear term $C^{h}(\cdot ; \cdot, \cdot)$ (see $\left.(3.17)\right)$, we have that

$$
\begin{aligned}
& C\left(\psi ; \psi, \phi_{h}\right)-C^{h}\left(\psi ; \psi, \phi_{h}\right) \\
&=\sum_{K \in \mathcal{T}_{h}}\left(\int_{K} \Delta \psi \operatorname{curl} \psi \cdot \nabla \phi_{h}-\int_{K}\left(\Delta \Pi_{K}^{k, \Delta} \psi\right) \Pi_{K}^{k-1} \operatorname{curl} \psi \cdot \Pi_{K}^{k-1} \nabla \phi_{h}\right), \\
&=\sum_{K \in \mathcal{T}_{h}}\left(\int_{K} \Delta \psi \operatorname{curl} \psi \cdot\left(I-\Pi_{K}^{k-1}\right)\left(\nabla \phi_{h}\right)+\int_{K} \Delta \psi\left(I-\Pi_{K}^{k-1}\right) \operatorname{curl} \psi \cdot \Pi_{K}^{k-1} \nabla \phi_{h}\right. \\
&\left.\quad+\int_{K} \Delta\left(I-\Pi_{K}^{k, \Delta}\right) \psi \Pi_{K}^{k-1} \operatorname{curl} \psi \cdot \Pi_{K}^{k-1} \nabla \phi_{h}\right) \\
&=: D_{1}+D_{2}+D_{3} .
\end{aligned}
$$


Next, we bound the term $D_{1}$, and we split it in two cases. First, we consider the case $1 / 2<r \leq 1$.

$$
\begin{aligned}
D_{1} & =\sum_{K \in \mathcal{T}_{h}} \int_{K} \Delta \psi \operatorname{curl} \psi \cdot\left(I-\mathbf{\Pi}_{K}^{k-1}\right) \nabla \phi_{h} \\
& \leq \sum_{K \in \mathcal{T}_{h}}\|\Delta \psi\|_{L^{4}(K)}\|\operatorname{curl} \psi\|_{L^{4}(K)}\left\|\left(I-\Pi_{K}^{k-1}\right) \nabla \phi_{h}\right\|_{0, K} \\
& \leq C h\left(\sum_{K \in \mathcal{T}_{h}}\|\Delta \psi\|_{L^{4}(K)}^{4}\right)^{1 / 4}\left(\sum_{K \in \mathcal{T}_{h}}\|\operatorname{curl} \psi\|_{L^{4}(K)}^{4}\right)^{1 / 4}\left(\sum_{K \in \mathcal{T}_{h}}\left|\phi_{h}\right|_{2, K}^{2}\right)^{1 / 2} \\
& \leq C h\|\Delta \psi\|_{L^{4}(\Omega)}\|\operatorname{curl} \psi\|_{L^{4}(\Omega)}\left|\phi_{h}\right|_{2, \Omega} \\
& \leq C h\|\psi\|_{2+r, \Omega}\|\operatorname{curl} \psi\|_{r, \Omega}\left|\phi_{h}\right|_{2, \Omega} \\
& \leq C h\|\psi\|_{2+r, \Omega}\|\psi\|_{1+r, \Omega}\left|\phi_{h}\right|_{2, \Omega}
\end{aligned}
$$

where we have used the Sobolev embedding $H^{r}(\Omega) \hookrightarrow L^{4}(\Omega)$ for $r \in(1 / 2,1]$. On the other hand, for the case $1 \leq r \leq k-1$, we proceed as follows. Using the orthogonality property of the projection operator $\mathbf{\Pi}_{K}^{k-1}$ on the polynomial function of degree $k-1$ and Cauchy-Schwarz inequality, we bound the first term on the right hand side of (5.13).

$$
\begin{aligned}
D_{1}=\sum_{K \in \mathcal{T}_{h}} \int_{K} \Delta \psi \operatorname{curl} \psi \cdot\left(I-\mathbf{\Pi}_{K}^{k-1}\right) \nabla \phi_{h} & =\sum_{K \in \mathcal{T}_{h}} \int_{K}\left(I-\mathbf{\Pi}_{K}^{k-1}\right)(\Delta \psi \operatorname{curl} \psi)\left(I-\mathbf{\Pi}_{K}^{k-1}\right) \nabla \phi_{h} \\
& \leq \sum_{K \in \mathcal{T}_{h}}\left\|\left(I-\mathbf{\Pi}_{K}^{k-1}\right)(\Delta \psi \operatorname{curl} \psi)\right\|_{0, K}\left\|\left(I-\mathbf{\Pi}_{K}^{k-1}\right) \nabla \phi_{h}\right\|_{0, K} \\
& \leq C h^{r-1}|(\Delta \psi \operatorname{curl} \psi)|_{r-1, \Omega} \tilde{C} h\left|\phi_{h}\right|_{2, \Omega} .
\end{aligned}
$$

An application of Hölder's inequality and Sobolev embedding theorem Lemma 4.2 of [9] yields,

$$
|\Delta \psi \operatorname{curl} \psi|_{r-1, \Omega} \leq C\|\Delta \psi\|_{W^{r-1,4}(\Omega)}\|\operatorname{curl} \psi\|_{W^{r-1,4}(\Omega)} \leq C\|\psi\|_{2+r, \Omega}\|\psi\|_{1+r, \Omega}
$$

Collecting the above inequalities, we obtain for $r>1 / 2$ that

$$
D_{1}=\sum_{K \in \mathcal{T}_{h}} \int_{K} \Delta \psi \operatorname{curl} \psi \cdot\left(\left(I-\mathbf{\Pi}_{K}^{k-1}\right) \nabla \phi_{h}\right) \mid \leq C h^{r}\|\psi\|_{1+r, \Omega}\|\psi\|_{2+r, \Omega}\left\|\phi_{h}\right\|_{2, \Omega}
$$

Using Hölder's inequality, the term $D_{2}$ in (5.13) can be bounded as follows

$$
\sum_{K \in \mathcal{T}_{h}} \int_{K} \Delta \psi\left(\left(I-\boldsymbol{\Pi}_{K}^{k-1}\right) \operatorname{curl} \psi\right) \cdot \boldsymbol{\Pi}_{K}^{k-1} \nabla \phi_{h} \leq \sum_{K \in \mathcal{T}_{h}} C\|\Delta \psi\|_{0, K}\left\|\left(I-\boldsymbol{\Pi}_{K}^{k-1}\right) \operatorname{curl} \psi\right\|_{L^{4}(K)}\left\|\mathbf{\Pi}_{K}^{k-1} \nabla \phi_{h}\right\|_{L^{4}(K)} .
$$

Using the continuity of $\Pi_{K}^{k-1}$ on the space $L^{4}(K)$ and optimal approximation property of the polynomial projection operator, we have

$$
\begin{aligned}
\left\|\left(I-\boldsymbol{\Pi}_{K}^{k-1}\right) \operatorname{curl} \psi\right\|_{L^{4}(K)} & \leq\left\|\operatorname{curl}\left(\psi-\psi_{\pi}\right)\right\|_{L^{4}(K)}+\left\|\boldsymbol{\Pi}_{K}^{k-1}\left(\operatorname{curl} \psi-\operatorname{curl} \psi_{\pi}\right)\right\|_{L^{4}(K)} \\
& \leq C h^{r}|\psi|_{W^{r, 4}(K)} .
\end{aligned}
$$

Also, the term can be bounded as

$$
\left\|\boldsymbol{\Pi}_{K}^{k-1} \nabla \phi_{h}\right\|_{L^{4}(\Omega)} \leq\left\|\nabla \phi_{h}\right\|_{L^{4}(\Omega)} \leq C\left|\phi_{h}\right|_{2, \Omega},
$$


where we have used that $H^{1}(\Omega) \hookrightarrow L^{4}(\Omega)$. Hence, by the Sobolev embedding $H^{1+r}(\Omega) \hookrightarrow W^{r, 4}(\Omega)$, we can write

$$
D_{2} \leq \sum_{K \in \mathcal{T}_{h}}\left|\int_{K} \Delta \psi\left(\left(I-\boldsymbol{\Pi}_{K}^{k-1}\right) \operatorname{curl} \psi\right) \cdot \boldsymbol{\Pi}_{K}^{k-1} \nabla \phi_{h}\right| \leq C h^{r}\|\Delta \psi\|_{0, \Omega}|\psi|_{2+r, \Omega}\left|\phi_{h}\right|_{2, \Omega} .
$$

Using the Hölder's inequality and suming over each element $K$, the third term of (5.13) can be bounded as follows

$$
\begin{aligned}
D_{3} & =\sum_{K \in \mathcal{T}_{h}} \int_{K} \Delta\left(I-\Pi_{K}^{k, \Delta}\right) \psi \boldsymbol{\Pi}_{K}^{k-1} \operatorname{curl} \psi \cdot \Pi_{K}^{k-1} \nabla \phi_{h} \\
& \leq \sum_{K \in \mathcal{T}_{h}} \int_{K}\left|\left(I-\Pi_{K}^{k, \Delta}\right) \psi\right|_{2, K}\left\|\boldsymbol{\Pi}_{K}^{k-1} \operatorname{curl} \psi\right\|_{L^{4}(K)}\left\|\boldsymbol{\Pi}_{K}^{k-1} \nabla \phi_{h}\right\|_{L^{4}(K)} .
\end{aligned}
$$

Therefore, from the fact that $\Pi_{K}^{k, \Delta}$ is the projector defined by (3.1a), the continuity of $\Pi_{K}^{k-1}$ on the space $L^{4}(K)$ and Sobolev's embedding theorem, we obtain

$$
D_{3} \leq C h^{r}|\psi|_{2+r, \Omega}|\psi|_{2, \Omega}\left|\phi_{h}\right|_{2, \Omega} .
$$

Finally, the proof follows by collecting all the estimations (5.14)-(5.16) and inserting into (5.13).

The following theorem provides the error estimates for the semi-discrete virtual element scheme.

Theorem 5.5. Let $\psi(t) \in W$ be the solution of problem (2.5) and let $\psi_{h}(t) \in W_{h}$ be the solution of problem (4.1). Assume that $\psi(t) \in H^{2+r}(\Omega), \partial_{t} \psi(t) \in H^{1+r}(\Omega)$, for $\frac{1}{2}<r \leq k-1$ and for almost all $t \in[0, T]$. In addition, assume that $\psi, \psi_{h} \in \mathcal{K}:=\left\{v \in W: \frac{2\|v\|_{2, \Omega}}{\alpha_{3} \nu}<1\right\}$. Then, there exists a positive generic constant $C$, which could be depended on mesh regularity Assumption 3.1, Sobolev regularity of the solution $\psi, \partial_{t} \psi, \partial_{t t} \psi$ but independents of mesh size $h$ such that the following estimation holds

$$
\begin{aligned}
& \left\|\psi-\psi_{h}\right\|_{L^{\infty}\left(0, t, H^{1}(\Omega)\right)}+C\left\|\psi-\psi_{h}\right\|_{L^{2}\left(0, t, H^{2}(\Omega)\right)} \leq\left\|\psi(0)-\psi_{h}(0)\right\|_{1, \Omega}+C\left(C_{A}\right) h^{r}\left(\|\psi(0)\|_{1+r, \Omega}\right. \\
& \left.\quad+\|\mathbf{f}\|_{L^{2}\left(0, t, H^{r-1}(\Omega)\right)}+\left\|\partial_{t} \psi\right\|_{L^{2}\left(0, t, H^{1+r}(\Omega)\right)}+\|\psi\|_{L^{2}\left(0, t, H^{2+r}(\Omega)\right)}\right) .
\end{aligned}
$$

Proof. Upon applying the projection operator $\mathcal{S}_{h}$, we split the error as (see (5.2)):

$$
\begin{aligned}
\psi_{h}-\psi & :=\psi_{h}-\mathcal{S}_{h} \psi+\mathcal{S}_{h} \psi-\psi \\
& =: \theta_{h}+\rho_{h} .
\end{aligned}
$$

Since the estimation for $\rho_{h}$ is known from the Lemma 5.3, we attempt to bound $\theta_{h}$.

Using the semi-discrete scheme (4.1), definition of the projection operator $\mathcal{S}_{h}(5.1)$ and the continuous weak formulation (2.3), we obtain

$$
\begin{aligned}
A^{h}\left(\partial_{t} \theta_{h}(t), \phi_{h}\right)+\nu B^{h}\left(\theta_{h}(t), \phi_{h}\right)= & F^{h}\left(\phi_{h}\right)-C^{h}\left(\psi_{h} ; \psi_{h}, \phi_{h}\right)-A^{h}\left(\partial_{t} \mathcal{S}_{h} \psi, \phi_{h}\right)-\nu B^{h}\left(\mathcal{S}_{h} \psi, \phi_{h}\right) \\
= & F^{h}\left(\phi_{h}\right)-F\left(\phi_{h}\right)-C^{h}\left(\psi_{h} ; \psi_{h}, \phi_{h}\right)+C\left(\psi ; \psi, \phi_{h}\right) \\
& -A^{h}\left(\partial_{t} \mathcal{S}_{h} \psi, \phi_{h}\right)+A\left(\partial_{t} \psi, \phi_{h}\right)-\nu B^{h}\left(\mathcal{S}_{h} \psi, \phi_{h}\right)+\nu B\left(\psi, \phi_{h}\right) .
\end{aligned}
$$

Now, we will bound all the terms on the right hand side above. We start with the nonlinear terms $C\left(\psi ; \psi, \phi_{h}\right)-$ $C^{h}\left(\psi_{h} ; \psi_{h}, \phi_{h}\right)$. We rewrite the term as follows:

$$
C\left(\psi ; \psi, \phi_{h}\right)-C^{h}\left(\psi_{h} ; \psi_{h}, \phi_{h}\right)=\underbrace{C\left(\psi ; \psi, \phi_{h}\right)-C^{h}\left(\psi ; \psi, \phi_{h}\right)}_{=: A_{1}}+\underbrace{C^{h}\left(\psi ; \psi, \phi_{h}\right)-C^{h}\left(\psi_{h} ; \psi_{h}, \phi_{h}\right)}_{=: A_{2}} .
$$


The term $A_{1}$ has been bounded in Lemma 5.4. Thus, we will bound the term $A_{2}$.

We rewrite the term $A_{2}$ as follows

$$
\begin{aligned}
C^{h}\left(\psi ; \psi, \phi_{h}\right)-C^{h}\left(\psi_{h} ; \psi_{h}, \phi_{h}\right) \\
=\sum_{K \in \mathcal{T}_{h}}\left(\int_{K} \Delta \Pi_{K}^{k, \Delta} \psi \Pi_{K}^{k-1} \operatorname{curl} \psi \cdot \Pi_{K}^{k-1} \operatorname{curl} \phi_{h}-\int_{K} \Delta \Pi_{K}^{k, \Delta} \psi_{h} \Pi_{K}^{k-1} \operatorname{curl} \psi_{h} \cdot \Pi_{K}^{k-1} \operatorname{curl} \phi_{h}\right), \\
=\sum_{K \in \mathcal{T}_{h}}\left(\int_{K} \Delta \Pi_{K}^{k, \Delta} \psi \Pi_{K}^{k-1} \operatorname{curl} \psi \cdot \Pi_{K}^{k-1} \operatorname{curl} \phi_{h}-\int_{K} \Delta \Pi_{K}^{k, \Delta} \psi_{h} \Pi_{K}^{k-1} \operatorname{curl} \psi \cdot \Pi_{K}^{k-1} \operatorname{curl} \phi_{h}\right. \\
\left.\quad+\int_{K} \Delta \Pi_{K}^{k, \Delta} \psi_{h} \Pi_{K}^{k-1} \operatorname{curl} \psi \cdot \Pi_{K}^{k-1} \operatorname{curl} \phi_{h}-\int_{K} \Delta \Pi_{K}^{k, \Delta} \psi_{h} \Pi_{K}^{k-1} \operatorname{curl} \psi_{h} \cdot \Pi_{K}^{k-1} \operatorname{curl} \phi_{h}\right) \\
=\sum_{K \in \mathcal{T}_{h}}\left(\int_{K} \Delta\left(\Pi_{K}^{k, \Delta} \psi-\Pi_{K}^{k, \Delta} \psi_{h}\right) \Pi_{K}^{k-1} \operatorname{curl} \psi \cdot \Pi_{K}^{k-1} \operatorname{curl} \phi_{h}\right. \\
\left.\quad+\int_{K} \Delta \Pi_{K}^{k, \Delta} \psi_{h} \Pi_{K}^{k-1}\left(\operatorname{curl} \psi-\operatorname{curl} \psi_{h}\right) \cdot \Pi_{K}^{k-1} \operatorname{curl} \phi_{h}\right)
\end{aligned}
$$

Using Hölder's inequality, the first term on the right hand side of (5.18) can be bounded as follows,

$$
\begin{aligned}
& \sum_{K \in \mathcal{T}_{h}} \int_{K} \Delta\left(\Pi_{K}^{k, \Delta} \psi-\Pi_{K}^{k, \Delta} \psi_{h}\right) \Pi_{K}^{k-1} \operatorname{curl} \psi \cdot \Pi_{K}^{k-1} \operatorname{curl} \phi_{h} \\
& \quad \leq C \sum_{K \in \mathcal{T}_{h}}\left\|\Delta \Pi_{K}^{k, \Delta}\left(\psi-\psi_{h}\right)\right\|_{0, K}\left\|\boldsymbol{\Pi}_{K}^{k-1} \operatorname{curl} \psi\right\|_{L^{4}(K)}\left\|\boldsymbol{\Pi}_{K}^{k-1} \operatorname{curl} \phi_{h}\right\|_{L^{4}(K)} \\
& \quad \leq C\left\|\Delta \Pi_{K}^{k, \Delta}\left(\psi-\psi_{h}\right)\right\|_{0, \Omega}\left\|\Pi_{K}^{k-1} \operatorname{curl} \psi\right\|_{L^{4}(\Omega)}\left\|\Pi_{K}^{k-1} \operatorname{curl} \phi_{h}\right\|_{L^{4}(\Omega)} \\
& \quad \leq C\left|\psi-\psi_{h}\right|_{2, \Omega}\|\operatorname{curl} \psi\|_{1, \Omega}\left\|\operatorname{curl} \phi_{h}\right\|_{1, \Omega} \\
& \quad \leq C\left(\left|\psi-\mathcal{S}_{h} \psi\right|_{2, \Omega}+\left|\mathcal{S}_{h} \psi-\psi_{h}\right|_{2, \Omega}\right)\|\operatorname{curl} \psi\|_{1, \Omega}\left\|\operatorname{curl} \phi_{h}\right\|_{1, \Omega} \\
& \quad \leq C\left(h^{r}|\psi|_{2+r, \Omega}+\left|\theta_{h}(t)\right|_{2, \Omega}\right)\|\psi\|_{2, \Omega}\left\|\phi_{h}\right\|_{2, \Omega}
\end{aligned}
$$

where we have used the boundedness of $\Pi_{K}^{k, \Delta}$, Sobolev's embedding theorem and the approximation property of the operator $\mathcal{S}_{h}(c f .(5.3))$.

Using Hölder's inequality, we can bound the second term on the right hand side of (5.18) as follows,

$$
\begin{aligned}
& \sum_{K \in \mathcal{T}_{h}} \int_{K} \Delta\left(\Pi_{K}^{k, \Delta} \psi_{h}\right) \Pi_{K}^{k-1}\left(\operatorname{curl} \psi-\operatorname{curl} \psi_{h}\right) \Pi_{K}^{k-1} \nabla \phi_{h} \\
& \quad \leq \sum_{K \in \mathcal{T}_{h}}\left\|\Delta \Pi_{K}^{k, \Delta} \psi_{h}\right\|_{L^{2}(K)}\left\|\Pi_{K}^{k-1}\left(\operatorname{curl} \psi-\operatorname{curl} \psi_{h}\right)\right\|_{L^{4}(K)}\left\|\mathbf{\Pi}_{K}^{k-1} \nabla \phi_{h}\right\|_{L^{4}(K)} \\
& \quad \leq C\left|\psi_{h}\right|_{2, \Omega}\left\|\operatorname{curl} \psi-\operatorname{curl} \psi_{h}\right\|_{L^{4}(\Omega)}\left\|\nabla \phi_{h}\right\|_{L^{4}(\Omega)} \\
& \quad \leq C\left|\psi_{h}\right|_{2, \Omega}\left\|\operatorname{curl} \psi-\operatorname{curl} \psi_{h}\right\|_{1, \Omega}\left\|\nabla \phi_{h}\right\|_{1, \Omega} \\
& \quad \leq C\left|\psi_{h}\right|_{2, \Omega}\left(h^{r}|\psi|_{2+r, \Omega}+\left|\theta_{h}(t)\right|_{2, \Omega}\right)\left\|\phi_{h}\right\|_{2, \Omega}
\end{aligned}
$$

where we have exploited Sobolev inequality and approximation property of $\mathcal{S}_{h}$ (cf. (5.3)). 
Using the polynomial consistency property of discrete bilinear form $A^{h}(\cdot, \cdot)(c f .(3.10))$, we can represent the time derivative term in (5.17) as

$$
\begin{aligned}
-A^{h}\left(\partial_{t} \mathcal{S}_{h} \psi, \phi_{h}\right)+A\left(\partial_{t} \psi, \phi_{h}\right)= & A^{h}\left(-\mathcal{S}_{h} \partial_{t} \psi, \phi_{h}\right)+A\left(\partial_{t} \psi, \phi_{h}\right) \\
& +\sum_{K \in \mathcal{T}_{h}}\left(A_{K}^{h}\left(\Pi_{K}^{k, \nabla^{\perp}} \partial_{t} \psi, \phi_{h}\right)-A_{K}\left(\Pi_{K}^{k, \nabla^{\perp}} \partial_{t} \psi, \phi_{h}\right)\right) \\
= & \sum_{K \in \mathcal{T}_{h}} A_{K}^{h}\left(\Pi_{K}^{k, \nabla^{\perp}} \partial_{t} \psi-\mathcal{S}_{h} \partial_{t} \psi, \phi_{h}\right)+A_{K}\left(\partial_{t} \psi-\Pi_{K}^{k, \nabla^{\perp}} \partial_{t} \psi, \phi_{h}\right) \\
\leq & C \sum_{K \in \mathcal{T}_{h}}\left|\Pi_{K}^{k, \nabla^{\perp}} \partial_{t} \psi-\mathcal{S}_{h} \partial_{t} \psi\right|_{1, K}\left|\phi_{h}\right|_{1, K}+\left.\left.\left|\partial_{t} \psi-\Pi_{K}^{k, \nabla^{\perp}} \partial_{t} \psi\right|_{1, K}\right|_{h}\right|_{1, K} \\
\leq & C\left(C_{A}\right) h^{r}\left|\partial_{t} \psi\right|_{1+r, \Omega}\left|\phi_{h}\right|_{2, \Omega},
\end{aligned}
$$

where we have used the Cauchy-Schwarz inequality, the approximation property of the projection operator $\Pi_{K}^{k, \nabla^{\perp}}$ and Lemma 5.3 .

Now, we move to bound the load term in (5.17). To bound this term ( $c f .(3.15))$, we will exploit the approximation property of the projection operator $\boldsymbol{\Pi}_{K}^{k-1}$, which implies

$$
\begin{aligned}
\left|F^{h}\left(\phi_{h}\right)-F\left(\phi_{h}\right)\right| & =\sum_{K \in \mathcal{T}_{h}}\left|\int_{K}\left(\mathbf{\Pi}_{K}^{k-1} \mathbf{f}-\mathbf{f}\right) \cdot \operatorname{curl} \phi_{h}\right| \\
& \leq \sum_{K \in \mathcal{T}_{h}}\left\|\mathbf{\Pi}_{K}^{k-1} \mathbf{f}-\mathbf{f}\right\|_{0, K}\left\|\mathbf{\Pi}_{K}^{k-1} \operatorname{curl} \phi_{h}-\operatorname{curl} \phi_{h}\right\|_{0, K} \\
& \leq C h^{r}|\mathbf{f}|_{r-1, \Omega}\left|\phi_{h}\right|_{2, \Omega} .
\end{aligned}
$$

Now, inserting the estimations (5.18), (5.12), (5.19), (5.20), (5.21) into (5.17), choosing test function $\phi_{h}=$ $\theta_{h}(t)$, using stability properties of the bilinear forms $A^{h}(\cdot, \cdot)$ and $B^{h}(\cdot, \cdot)$, we derive

$$
\begin{aligned}
\alpha_{1} \frac{1}{2} \frac{\mathrm{d}}{\mathrm{d} t}\left\|\operatorname{curl} \theta_{h}(t)\right\|_{0, \Omega}^{2}+\alpha_{3} \nu\left\|\theta_{h}(t)\right\|_{2, \Omega}^{2} \leq & C h^{r}|\mathbf{f}|_{r-1, \Omega}\left\|\theta_{h}(t)\right\|_{2, \Omega}+C\left(C_{A}\right) h^{r}\left|\partial_{t} \psi\right|_{1+r, \Omega}\left\|\theta_{h}(t)\right\|_{2, \Omega} \\
& +C\left(h^{r}|\psi|_{2+r, \Omega}+\left\|\theta_{h}(t)\right\|_{2, \Omega}\right)\|\psi\|_{2, \Omega}\left\|\theta_{h}(t)\right\|_{2, \Omega} \\
& +\left\|\psi_{h}\right\|_{2, \Omega}\left(h^{r}|\psi|_{2+r, \Omega}+\left\|\theta_{h}(t)\right\|_{2, \Omega}\right)\left\|\theta_{h}(t)\right\|_{2, \Omega} .
\end{aligned}
$$

Upon applying kick-back argument, we obtain

$$
\begin{gathered}
\alpha_{1} \frac{1}{2} \frac{\mathrm{d}}{\mathrm{d} t}\left\|\operatorname{curl} \theta_{h}(t)\right\|_{0, \Omega}^{2}+\left(\alpha_{3} \nu-\|\psi\|_{2, \Omega}-\left\|\psi_{h}\right\|_{2, \Omega}\right)\left\|\theta_{h}(t)\right\|_{2, \Omega}^{2} \\
\leq C\left(C_{A}\right) h^{r}\left(|\mathbf{f}|_{r-1, \Omega}+\left|\partial_{t} \psi\right|_{1+r, \Omega}+|\psi|_{2+r, \Omega}\right)\left\|\theta_{h}(t)\right\|_{2, \Omega} .
\end{gathered}
$$

Since $\psi, \psi_{h} \in \mathcal{K}$, from the assumption of the theorem, and applying Young's inequality and taking integration on both sides of (5.22), we derive

$$
\begin{aligned}
\left\|\operatorname{curl} \theta_{h}(t)\right\|_{0, \Omega}+C\left(\alpha_{3}, \nu, \mathcal{R}\right)\left\|\theta_{h}(t)\right\|_{L^{2}\left(0, t, H^{2}(\Omega)\right)} \leq & \left\|\operatorname{curl} \theta_{h}(0)\right\|_{0, \Omega}+C\left(C_{A}\right) h^{r}\left(|\mathbf{f}|_{L^{2}\left(0, t, H^{r-1}(\Omega)\right)}\right. \\
& \left.+\left|\partial_{t} \psi\right|_{L^{2}\left(0, t, H^{1+r}(\Omega)\right)}+|\psi|_{L^{2}\left(0, t, H^{2+r}(\Omega)\right)}\right),
\end{aligned}
$$

for almost all $t \in(0, T]$. Using the approximation properties of $\mathcal{S}_{h}$ (Lem. 5.3), we derive

$$
\begin{aligned}
\left\|\psi-\psi_{h}\right\|_{L^{\infty}\left(0, t, H^{1}(\Omega)\right)}+C\left(\alpha_{3}, \nu, \mathcal{R}\right) \| \psi & -\psi_{h}\left\|_{L^{2}\left(0, t, H^{2}(\Omega)\right)} \leq\right\| \psi(0)-\psi_{h}(0) \|_{1, \Omega}+C\left(C_{A}\right) h^{r}\left(|\psi(0)|_{r+1, \Omega}\right. \\
& \left.+|\mathbf{f}|_{L^{2}\left(0, t, H^{r-1}(\Omega)\right)}+\left|\partial_{t} \psi\right|_{L^{2}\left(0, t, H^{1+r}(\Omega)\right)}+|\psi|_{L^{2}\left(0, t, H^{2+r}(\Omega)\right)}\right) .
\end{aligned}
$$

The proof is complete. 


\subsection{Error estimation for fully-discrete scheme}

In this section, we would like to derive the error estimation for the fully-discrete scheme $(c f .(4.7))$. To derive the estimates, we first split the error as follows:

$$
\psi_{h}^{n}-\psi\left(t_{n}\right)=\psi_{h}^{n}-\mathcal{S}_{h} \psi\left(t_{n}\right)+\mathcal{S}_{h} \psi\left(t_{n}\right)-\psi\left(t_{n}\right)=\theta_{h}^{n}+\rho_{h}^{n},
$$

where $\theta_{h}^{n}:=\psi_{h}^{n}-\mathcal{S}_{h} \psi\left(t_{n}\right)$ and $\rho_{h}^{n}:=\mathcal{S}_{h} \psi\left(t_{n}\right)-\psi\left(t_{n}\right)$. Since the estimation for $\rho_{h}^{n}$ is known from the Lemma 5.3, we will focus in bounding the term $\theta_{h}^{n}$.

Theorem 5.6. Let $\psi_{h}^{n} \in W_{h}$ be the virtual element solution generated by (4.7), and $\psi\left(t_{n}\right) \in W$ be the analytical solution of the problem (2.3) at time $t=t_{n}$. Assume that $\psi\left(t_{n}\right), \psi_{h}^{n} \in \widetilde{\mathcal{K}}:=\left\{v \in \mathcal{B}_{\mathcal{R}}: \frac{2 \mathcal{R}}{\alpha_{3} \nu}<1\right\}$. Also, we assume that the Assumption 3.1 is satisfied on mesh regularity. Then, under the condition of the Theorem 5.5, there exists a positive generic constant $C$ that depends on mesh regularity parameter, and Sobolev regularity of the exact solution $\psi, \partial_{t} \psi, \partial_{t t} \psi$ and force function $\mathbf{f}$ but independent of mesh size $h$ and time steps $\Delta t$ such that the following estimation holds

$$
\left\|\psi_{h}^{n}-\psi\left(t_{n}\right)\right\|_{1, \Omega}+\left(\Delta t \sum_{j=1}^{n}\left\|\psi_{h}^{j}-\psi\left(t_{j}\right)\right\|_{2, \Omega}^{2}\right)^{1 / 2} \leq C\left(C_{A}, \alpha_{3}, \mathcal{R}, \psi, \partial_{t} \psi, \partial_{t t} \psi, \mathbf{f}\right)\left(h^{r}+\Delta t\right),
$$

for $\frac{1}{2}<r \leq k-1$.

Proof. Using the fully discrete scheme (4.7), weak formulation (2.3), and the biharmonic projection operator $\mathcal{S}_{h}$, we obtain

$$
\begin{aligned}
A^{h}\left(\frac{\theta_{h}^{n}-\theta_{h}^{n-1}}{\Delta t}, \phi_{h}\right)+\nu B^{h}\left(\theta_{h}^{n}, \phi_{h}\right)= & A^{h}\left(\frac{\psi_{h}^{n}-\psi_{h}^{n-1}}{\Delta t}, \phi_{h}\right)+\nu B^{h}\left(\psi_{h}^{n}, \phi_{h}\right) \\
& -A^{h}\left(\frac{\mathcal{S}_{h} \psi\left(t_{n}\right)-\mathcal{S}_{h} \psi\left(t_{n-1}\right)}{\Delta t}, \phi_{h}\right)-\nu B^{h}\left(\mathcal{S}_{h} \psi\left(t_{n}\right), \phi_{h}\right) \\
= & F^{h}\left(\phi_{h}\right)-F\left(\phi_{h}\right)-C^{h}\left(\psi_{h}^{n} ; \psi_{h}^{n}, \phi_{h}\right)+C\left(\psi\left(t_{n}\right) ; \psi\left(t_{n}\right), \phi_{h}\right) \\
& +A\left(\partial_{t} \psi\left(t_{n}\right), \phi_{h}\right)-A^{h}\left(\frac{\mathcal{S}_{h} \psi\left(t_{n}\right)-\mathcal{S}_{h} \psi\left(t_{n-1}\right)}{\Delta t}, \phi_{h}\right) .
\end{aligned}
$$

In order to derive the desired estimation, we will put $\phi_{h}=\theta_{h}^{n}$ into (5.23).

$$
\begin{aligned}
A^{h}\left(\frac{\theta_{h}^{n}-\theta_{h}^{n-1}}{\Delta t}, \theta_{h}^{n}\right)+\nu B^{h}\left(\theta_{h}^{n}, \theta_{h}^{n}\right)= & F^{h}\left(\theta_{h}^{n}\right)-F\left(\theta_{h}^{n}\right)-C^{h}\left(\psi_{h}^{n} ; \psi_{h}^{n}, \theta_{h}^{n}\right)+C\left(\psi\left(t_{n}\right) ; \psi\left(t_{n}\right), \theta_{h}^{n}\right) \\
& +A\left(\partial_{t} \psi\left(t_{n}\right), \theta_{h}^{n}\right)-A^{h}\left(\frac{\mathcal{S}_{h} \psi\left(t_{n}\right)-\mathcal{S}_{h} \psi\left(t_{n-1}\right)}{\Delta t}, \theta_{h}^{n}\right) .
\end{aligned}
$$

The nonlinear term can be rewritten as follows:

$$
\begin{aligned}
C\left(\psi\left(t_{n}\right) ; \psi\left(t_{n}\right), \theta_{h}^{n}\right)-C^{h}\left(\psi_{h}^{n} ; \psi_{h}^{n}, \theta_{h}^{n}\right)= & C\left(\psi\left(t_{n}\right) ; \psi\left(t_{n}\right), \theta_{h}^{n}\right)-C^{h}\left(\psi\left(t_{n}\right) ; \psi\left(t_{n}\right), \theta_{h}^{n}\right) \\
& +C^{h}\left(\psi\left(t_{n}\right) ; \psi\left(t_{n}\right), \theta_{h}^{n}\right)-C^{h}\left(\psi_{h}^{n} ; \psi_{h}^{n}, \theta_{h}^{n}\right) .
\end{aligned}
$$

Using analogous arguments as in the proof of the Theorem 5.5, we have

$$
\left|C\left(\psi\left(t_{n}\right) ; \psi\left(t_{n}\right), \theta_{h}^{n}\right)-C^{h}\left(\psi\left(t_{n}\right) ; \psi\left(t_{n}\right), \theta_{h}^{n}\right)\right| \leq C h^{r}\left|\psi\left(t_{n}\right)\right|_{2+r, \Omega}\left\|\psi\left(t_{n}\right)\right\|_{2, \Omega}\left\|\theta_{h}^{n}\right\|_{2, \Omega} .
$$


Proceeding same as semi-discrete case, another term of (5.25) can be estimated as follows

$$
\begin{aligned}
\left|C^{h}\left(\psi\left(t_{n}\right) ; \psi\left(t_{n}\right), \theta_{h}^{n}\right)-C^{h}\left(\psi_{h}^{n} ; \psi_{h}^{n} ; \theta_{h}^{n}\right)\right| \leq & C\left(h^{r}\left|\psi\left(t_{n}\right)\right|_{2+r, \Omega}+\left\|\Delta \theta_{h}^{n}\right\|_{0, \Omega}\right)\left\|\operatorname{curl} \psi\left(t_{n}\right)\right\|_{1, \Omega}\left\|\operatorname{curl} \theta_{h}^{n}\right\|_{1, \Omega} \\
& +\left\|\Delta \psi_{h}^{n}\right\|_{0, \Omega}\left(h^{r}\left|\psi\left(t_{n}\right)\right|_{2+r, \Omega}+\left\|\theta_{h}^{n}\right\|_{0, \Omega}\right)\left\|\nabla \theta_{h}^{n}\right\|_{1, \Omega} .
\end{aligned}
$$

Using the projection operator $\Pi_{K}^{k, \Delta}$, and polynomial consistency property of the discrete bilinear form $A^{h}(\cdot, \cdot)$, we obtain

$$
\begin{aligned}
& A\left(\psi_{t}\left(t_{n}\right), \theta_{h}^{n}\right)-A^{h}\left(\frac{\mathcal{S}_{h} \psi\left(t_{n}\right)-\mathcal{S}_{h} \psi\left(t_{n-1}\right)}{\Delta t}, \theta_{h}^{n}\right)=A\left(\psi_{t}\left(t_{n}\right)-\frac{\psi\left(t_{n}\right)-\psi\left(t_{n-1}\right)}{\Delta t}, \theta_{h}^{n}\right) \\
& \quad+\sum_{K \in \mathcal{T}_{h}} A_{K}\left(\frac{\psi\left(t_{n}\right)-\psi\left(t_{n-1}\right)}{\Delta t}-\left(\frac{\Pi_{K}^{k, \Delta}\left(\psi\left(t_{n}\right)-\psi\left(t_{n-1}\right)\right)}{\Delta t}\right), \theta_{h}^{n}\right) \\
& \quad+\sum_{K \in \mathcal{T}_{h}} A_{K}^{h}\left(\left(\frac{\Pi_{K}^{k, \Delta}\left(\psi\left(t_{n}\right)-\psi\left(t_{n-1}\right)\right)}{\Delta t}\right)-\frac{\mathcal{S}_{h} \psi\left(t_{n}\right)-\mathcal{S}_{h} \psi\left(t_{n-1}\right)}{\Delta t}, \theta_{h}^{n}\right) \\
& :=\Sigma_{1}+\Sigma_{2}+\Sigma_{3} .
\end{aligned}
$$

Following the analogous arguments as Theorem 3.3 of [40], we can write the term $\Sigma_{1}$ as follows,

$$
\begin{aligned}
\Sigma_{1} & \leq \frac{1}{\Delta t}\left|\psi\left(t_{n}\right)-\psi\left(t_{n-1}\right)-\Delta t \psi_{t}\left(t_{n}\right)\right|_{1, \Omega}\left|\theta_{h}^{n}\right|_{1, \Omega} \leq \frac{1}{\Delta t}\left|\int_{t_{n-1}}^{t_{n}}\left(s-t_{j-1}\right) \psi_{t t}(s)\right|_{1, \Omega}\left|\theta_{h}^{n}\right|_{1, \Omega} \\
& \leq\left\|\partial_{t t} \psi\right\|_{L^{1}\left(t_{n-1}, t_{n} ; L^{2}(\Omega)\right)}\left|\theta_{h}^{n}\right|_{1, \Omega} .
\end{aligned}
$$

Further, in order to bound $\Sigma_{2}$, we derive the following estimate

$$
\begin{aligned}
\Sigma_{2} & \leq \frac{1}{\Delta t} \sum_{K \in \mathcal{T}_{h}}\left\|\operatorname{curl}\left(\psi\left(t_{n}\right)-\psi\left(t_{n-1}\right)\right)-\operatorname{curl} \Pi_{K}^{k, \Delta}\left(\psi\left(t_{n}\right)-\psi\left(t_{n-1}\right)\right)\right\|_{0, K}\left|\theta_{h}^{n}\right|_{1, K} \\
& \leq \frac{1}{\Delta t} C h^{r}\left\|\partial_{t} \psi\right\|_{L^{1}\left(t_{n-1}, t_{n} ; H^{r+1}(\Omega)\right)}\left|\theta_{h}^{n}\right|_{1, \Omega} .
\end{aligned}
$$

Adding and subtracting $\operatorname{curl}\left(\psi\left(t_{n}\right)-\psi\left(t_{n-1}\right)\right)$, the term $\Sigma_{3}(c f .(5.28))$ can be written as

$$
\begin{aligned}
\Sigma_{3} \leq & \frac{1}{\Delta t} C_{A} \sum_{K \in \mathcal{T}_{h}}\left\|\operatorname{curl}\left(\mathcal{S}_{h} \psi\left(t_{n}\right)-\mathcal{S}_{h} \psi\left(t_{n-1}\right)\right)-\operatorname{curl} \Pi_{K}^{k, \Delta}\left(\psi\left(t_{n}\right)-\psi\left(t_{n-1}\right)\right)\right\|_{0, K}\left|\theta_{h}^{n}\right|_{1, K} \\
\leq & \frac{1}{\Delta t} C_{A} \sum_{K \in \mathcal{T}_{h}}\left\|\operatorname{curl}\left(\mathcal{S}_{h} \psi\left(t_{n}\right)-\mathcal{S}_{h} \psi\left(t_{n-1}\right)\right)-\operatorname{curl}\left(\psi\left(t_{n}\right)-\psi\left(t_{n-1}\right)\right)\right\|_{0, K}\left|\theta_{h}^{n}\right|_{1, K} \\
& +\left\|\operatorname{curl}\left(\psi\left(t_{n}\right)-\psi\left(t_{n-1}\right)\right)-\operatorname{curl} \Pi_{K}^{k, \Delta}\left(\psi\left(t_{n}\right)-\psi\left(t_{n-1}\right)\right)\right\|_{0, K}\left|\theta_{h}^{n}\right|_{1, K} .
\end{aligned}
$$

Using the approximation properties of the operator $\mathcal{S}_{h}$, we obtain

$$
\begin{aligned}
& \sum_{K \in \mathcal{T}_{h}}\left\|\operatorname{curl}\left(\mathcal{S}_{h} \psi\left(t_{n}\right)-\mathcal{S}_{h} \psi\left(t_{n-1}\right)\right)-\operatorname{curl}\left(\psi\left(t_{n}\right)-\psi\left(t_{n-1}\right)\right)\right\|_{0, K}\left|\theta_{h}^{n}\right|_{1, K} \\
& \quad \leq C h^{r}\left\|\partial_{t} \psi\right\|_{L^{1}\left(t_{n-1}, t_{n} ; H^{1+r}(\Omega)\right)}\left|\theta_{h}^{n}\right|_{1, \Omega} .
\end{aligned}
$$

Analogously, we have

$$
\begin{aligned}
& \sum_{K \in \mathcal{T}_{h}}\left\|\operatorname{curl}\left(\psi\left(t_{n}\right)-\psi\left(t_{n-1}\right)\right)-\operatorname{curl} \Pi_{K}^{k, \Delta}\left(\psi\left(t_{n}\right)-\psi\left(t_{n-1}\right)\right)\right\|_{0, K}\left|\theta_{h}^{n}\right|_{1, K} \\
& \quad \leq C h^{r}\left\|\partial_{t} \psi\right\|_{L^{1}\left(t_{n-1}, t_{n} ; H^{1+r}(\Omega)\right)}\left|\theta_{h}^{n}\right|_{1, \Omega} .
\end{aligned}
$$


Inserting the above estimates in (5.28), and then using it together with (5.26) and (5.27) into (5.24), and multiplying by $\Delta t$, we obtain that

$$
\begin{aligned}
& \alpha_{1}\left\|\operatorname{curl} \theta_{h}^{n}\right\|_{0, \Omega}^{2}+\nu \Delta t \alpha_{3}\left\|\theta_{h}^{n}\right\|_{2, \Omega}^{2} \leq C \Delta t h^{r}|\mathbf{f}|_{r-1, \Omega}\left\|\theta_{h}^{n}\right\|_{2, \Omega}+C \Delta t\left(h^{r}\left|\psi\left(t_{n}\right)\right|_{r+2, \Omega}+\left\|\theta_{h}^{n}\right\|_{2, \Omega}\right) \\
& \quad \times\left\|\psi\left(t_{n}\right)\right\|_{2, \Omega}\left\|\theta_{h}^{n}\right\|_{2, \Omega}+C \Delta t\left\|\psi_{h}^{n}\right\|_{2, \Omega}\left(h^{r}\left|\psi\left(t_{n}\right)\right|_{r+2, \Omega}+\left\|\theta_{h}^{n}\right\|_{2, \Omega}\right)\left\|\theta_{h}^{n}\right\|_{2, \Omega} \\
& \quad+C\left(C_{A}\right) \Delta t h^{r}\left\|\partial_{t} \psi\right\|_{L^{1}\left(t_{n-1}, t_{n} ; H^{r+1}(\Omega)\right)}\left\|\operatorname{curl} \theta_{h}^{n}\right\|_{0, \Omega}+C \Delta t\left\|\partial_{t t} \psi\right\|_{L^{1}\left(t_{n-1}, t_{n} ; L^{2}(\Omega)\right)}\left\|\operatorname{curl} \theta_{h}^{n}\right\|_{0, \Omega} \\
& \quad+\alpha_{1}\left\|\operatorname{curl} \theta_{h}^{n}\right\|_{0, \Omega}\left\|\operatorname{curl} \theta_{h}^{n-1}\right\|_{0, \Omega} .
\end{aligned}
$$

An application of the Young's inequality and kick-back argument yields

$$
\begin{aligned}
& \frac{\alpha_{1}}{2}\left\|\operatorname{curl} \theta_{h}^{n}\right\|_{0, \Omega}^{2}-\frac{\alpha_{1}}{2}\left\|\operatorname{curl} \theta_{h}^{n-1}\right\|_{0, \Omega}^{2}+\Delta t\left(\nu \alpha_{3}-\left\|\psi\left(t_{n}\right)\right\|_{2, \Omega}-\left\|\psi_{h}^{n}\right\|_{2, \Omega}\right)\left\|\theta_{h}^{n}\right\|_{2, \Omega}^{2} \\
& \leq \\
& \quad C h^{r}\left(|\mathbf{f}|_{r-1, \Omega}+\left|\psi\left(t_{n}\right)\right|_{r+2, \Omega}\right)\left\|\theta_{h}^{n}\right\|_{2, \Omega}+C\left(C_{A}\right) h^{r}\left\|\partial_{t} \psi\right\|_{L^{1}\left(t_{n-1}, t_{n} ; H^{r+1}(\Omega)\right)}\left\|\operatorname{curl} \theta_{h}^{n}\right\|_{0, \Omega} \\
& \quad+C \Delta t\left\|\partial_{t t} \psi\right\|_{L^{1}\left(t_{n-1}, t_{n} ; L^{2}(\Omega)\right)}\left\|\operatorname{curl} \theta_{h}^{n}\right\|_{0, \Omega} .
\end{aligned}
$$

Since $\psi\left(t_{n}\right), \psi_{h}^{n} \in \tilde{\mathcal{K}}$, from the assumption of the theorem, and using Young's inequality and iterating $j=$ $1, \ldots, n$, we have

$$
\begin{aligned}
& \frac{\alpha_{1}}{2}\left\|\operatorname{curl} \theta_{h}^{n}\right\|_{0, \Omega}^{2}+\Delta t C\left(\nu, \alpha_{3}, \mathcal{R}\right) \sum_{j=1}^{n}\left\|\theta_{h}^{j}\right\|_{2, \Omega}^{2} \leq \frac{\alpha_{1}}{2}\left\|\operatorname{curl} \theta_{h}^{0}\right\|_{0, \Omega}^{2}+C \Delta t^{2}\left\|\partial_{t t} \psi\right\|_{L^{1}\left(0, t_{n} ; L^{2}(\Omega)\right)}^{2} \\
& \quad+C\left(C_{A}\right) h^{2 r}\left(\|\mathbf{f}\|_{L^{\infty}\left(0, t_{n} ; H^{r-1}(\Omega)\right)}^{2}+\|\psi\|_{L^{\infty}\left(0, t_{n} ; H^{r+2}(\Omega)\right)}^{2}+\left\|\partial_{t} \psi\right\|_{L^{\infty}\left(0, t_{n} ; H^{r+1}(\Omega)\right)}^{2}\right) \\
& \leq C\left\|\psi_{h}^{0}-\mathcal{S}_{h}(\psi(0))\right\|_{1, \Omega}^{2}+C\left(C_{A}\right) h^{2 r}\left(\|\mathbf{f}\|_{L^{\infty}\left(0, t_{n} ; H^{r-1}(\Omega)\right)}^{2}+\|\psi\|_{L^{\infty}\left(0, t_{n} ; H^{r+2}(\Omega)\right)}^{2}\right. \\
&\left.+\left\|\partial_{t} \psi\right\|_{L^{\infty}\left(0, t_{n} ; H^{r+1}(\Omega)\right)}^{2}\right)+C \Delta t^{2}\left\|\partial_{t t} \psi\right\|_{L^{1}\left(0, t_{n} ; L^{2}(\Omega)\right)}^{2} \\
& \leq C\left(\left\|\psi_{I}(0)-\psi(0)\right\|_{1, \Omega}^{2}+\left\|\psi(0)-\mathcal{S}_{h}(\psi(0))\right\|_{1, \Omega}^{2}\right)+C \Delta t^{2}\left\|\partial_{t t} \psi\right\|_{L^{1}\left(0, t_{n} ; L^{2}(\Omega)\right)}^{2} \\
&+C\left(C_{A}\right) h^{2 r}\left(\|\mathbf{f}\|_{L^{\infty}\left(0, t_{n} ; H^{r-1}(\Omega)\right)}^{2}+\|\psi\|_{L^{\infty}\left(0, t_{n} ; H^{r+2}(\Omega)\right)}^{2}+\left\|\partial_{t} \psi\right\|_{L^{\infty}\left(0, t_{n} ; H^{r+1}(\Omega)\right)}^{2}\right)
\end{aligned}
$$

Using the approximation properties for $\mathcal{S}_{h}$, and Proposition 5.2, we get

$$
\begin{aligned}
\frac{\alpha_{1}}{2}\left\|\operatorname{curl} \theta_{h}^{n}\right\|_{0, \Omega}^{2} & +\Delta t C\left(\nu, \alpha_{3}, \mathcal{S}\right) \sum_{j=1}^{n}\left\|\theta_{h}^{j}\right\|_{2, \Omega}^{2} \leq C \Delta t^{2}\left\|\partial_{t t} \psi\right\|_{L^{1}\left(0, t_{n} ; L^{2}(\Omega)\right)}^{2} \\
& +C\left(C_{A}\right) h^{2 r}\left(\|\psi(0)\|_{2+r, \Omega}^{2}+\|\mathbf{f}\|_{L^{\infty}\left(0, t_{n} ; H^{r-1}(\Omega)\right)}^{2}+\|\psi\|_{L^{\infty}\left(0, t_{n} ; H^{r+2}(\Omega)\right)}^{2}\right. \\
& \left.+\left\|\partial_{t} \psi\right\|_{L^{\infty}\left(0, t_{n} ; H^{r+1}(\Omega)\right)}^{2}\right)
\end{aligned}
$$

Finally, using the fact that $\psi_{h}^{n}-\psi\left(t_{n}\right)=\theta_{h}^{n}+\rho_{h}^{n}$, from the above estimation and approximation properties for $\mathcal{S}_{h}$ (see Lem. 5.3), we have the desired thesis.

Remark 5.7. In Theorems 5.5 and 5.6, we have chosen that the analytical solution $\psi$ at time $t=t_{n}$ and fully discrete solution $\psi_{h}^{n}$ belong to a bounded subset of $\mathcal{B}_{\mathcal{R}}$. To satisfy this condition, an additional condition has to be imposed on viscosity $\nu$, which leads

$$
\max \left\{\frac{2 \hat{C}^{2}}{\alpha_{3}^{2} \nu^{2}}, \frac{2 C_{B}^{2}\left(4+\hat{C}^{2}\right)}{\alpha_{3}^{4} \nu^{2}}\right\}\left\|\psi_{h}\right\|_{L^{\infty}\left(0, t_{n-1} ; H^{2}(\Omega)\right)}^{2}<1 .
$$

For sufficiently small values of $\Delta t$ and $\nu$ satisfying the above mentioned condition, we advocate a numerical approximation of (2.3) that converges optimally in both space and time variable. The primary advantage of this scheme is that the condition imposed on $\nu$ is independent of $1 / \Delta t$ which ensures the robustness of the scheme for very small values of $\Delta t$. 

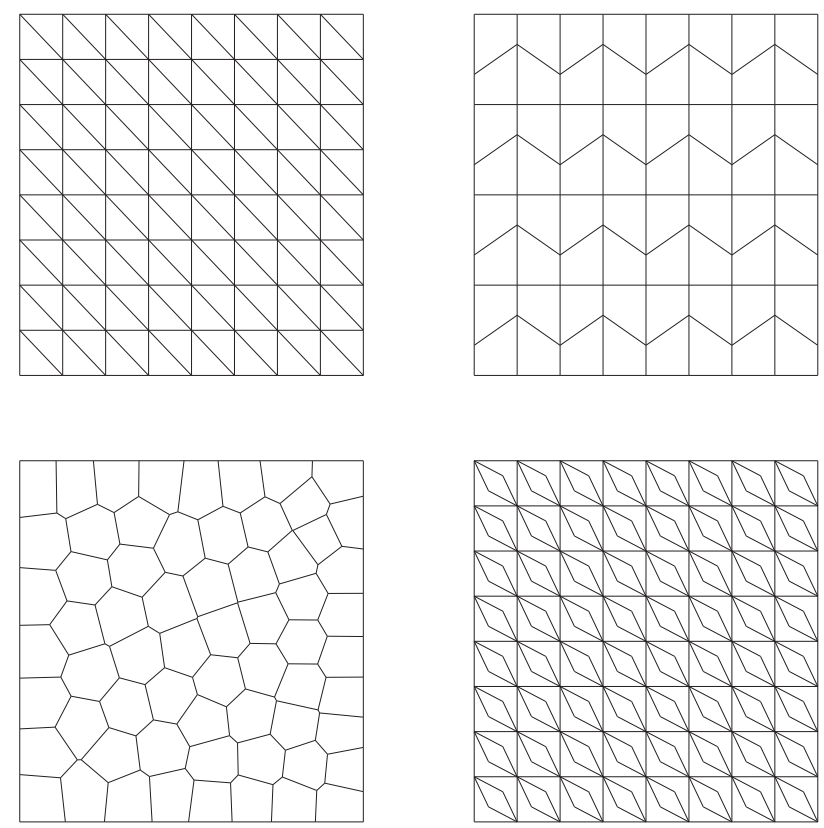

Figure 1. Sample meshes: $\mathcal{T}_{h}^{1}$ (top left), $\mathcal{T}_{h}^{2}$ (top right), $\mathcal{T}_{h}^{3}$ (bottom left) and $\mathcal{T}_{h}^{4}$ (bottom right).

\section{NUMERICAL EXPERIMENTS}

In this section, we report the results of four numerical tests carried out with the fully-discrete virtual element scheme proposed in Section 4, in order to validate the theoretical results presented in Section 5. We have developed a MATLAB code that implements the fully-discrete scheme for $k=2$ and $k=3$. For the time discretization, we employ a backward Euler scheme and for each time step, we use the Newton-Raphson method to solve the resulting nonlinear system, with maximum 10 iterations, a user specified tolerance tol: $=10^{-8}$, and taking $\psi_{h}^{\text {in }}=0$ as initial guess.

For our numerical tests, we have used different families of polygonal meshes (see Fig. 1):

$-\mathcal{T}_{h}^{1}$ : Uniform triangular meshes.

$-\mathcal{T}_{h}^{2}$ : Trapezoidal meshes.

$-\mathcal{T}_{h}^{3}$ : Sequence of Centroidal Voronoi Tessellation.

$-\mathcal{T}_{h}^{4}$ : Distorted concave rhombic quadrilaterals.

In order to test the convergence properties of the VEM method, we measure the errors as the difference between the exact solution $\psi$ and the suitable projections of the numerical solution $\psi_{h}$. More precisely, for the norm $L^{2}\left(0, T ; H^{2}(\Omega)\right)$, we consider the following quantity:

$$
\mathrm{E}_{2}(\psi):=\operatorname{error}\left(\psi, L^{2}, H^{2}\right)=\left(\Delta t \sum_{n=1}^{N}\left|\psi\left(t_{n}\right)-\Pi_{K}^{k, \Delta} \psi_{h}^{n}\right|_{2, h}^{2}\right)^{1 / 2}
$$

\subsection{Test 1. Homogeneous Dirichlet boundary conditions and initial data}

In this numerical test, we solve the Navier-Stokes problem $(2.1)$ on the square domain $\Omega:=(0,1)^{2}$. We take the load term $\mathbf{f}$, boundary and initial conditions in such a way that the analytical solution is given by:

$$
\boldsymbol{u}(x, y, t)=0.1\left(\begin{array}{c}
\sin (t) x^{2}(1-x)^{2}\left(2 y-6 y^{2}+4 y^{3}\right) \\
-\sin (t) y^{2}(1-y)^{2}\left(2 x-6 x^{2}+4 x^{3}\right)
\end{array}\right), \quad p(x, y, t)=t^{2}\left(x^{3} y^{3}-\frac{1}{16}\right)
$$


TABle 1. Test 1. Errors for the stream-function $\psi_{h}$ in the discrete $L^{2}\left(0, T ; H^{2}(\Omega)\right)$ norm obtained with $k=2, \nu=1$ and $\mathcal{T}_{h}^{2}$.

\begin{tabular}{|c|c|c|c|c|c|c|}
\hline dof & $h$ & $\Delta t_{0}$ & $\Delta t_{0} / 2$ & $\Delta t_{0} / 4$ & $\Delta t_{0} / 8$ & $\Delta t_{0} / 16$ \\
\hline 147 & $h_{0}$ & $1.076690 \mathrm{e}-3$ & $1.055725 \mathrm{e}-3$ & $1.045194 \mathrm{e}-3$ & $1.039916 \mathrm{e}-3$ & $1.037273 \mathrm{e}-3$ \\
\hline 675 & $h_{0} / 2$ & $5.450550 \mathrm{e}-4$ & \begin{tabular}{|l}
$5.344520 \mathrm{e}-4$ \\
\end{tabular} & $5.291258 \mathrm{e}-4$ & $5.264563 \mathrm{e}-4$ & \begin{tabular}{|l}
$5.251199 \mathrm{e}-4$ \\
\end{tabular} \\
\hline 2883 & $h_{0} / 4$ & $2.723653 \mathrm{e}-4$ & $2.670638 \mathrm{e}-4$ & $2.644015 \mathrm{e}-4$ & $2.630672 \mathrm{e}-4$ & $2.623994 \mathrm{e}-4$ \\
\hline 11907 & $h_{0} / 8$ & $1.361231 \mathrm{e}-4$ & $1.334675 \mathrm{e}-4$ & $1.321353 \mathrm{e}-4$ & \begin{tabular}{|l|}
$1.314681 \mathrm{e}-4$ \\
\end{tabular} & $1.311342 \mathrm{e}-4$ \\
\hline 48387 & $h_{0} / 16$ & $6.807611 \mathrm{e}-5$ & $6.673602 \mathrm{e}-5$ & $6.606687 \mathrm{e}-5$ & $6.573250 \mathrm{e}-5$ & $6.556536 \mathrm{e}-5$ \\
\hline
\end{tabular}

TABle 2. Test 1. Errors for the stream-function $\psi_{h}$ in the discrete $L^{2}\left(0, T ; H^{2}(\Omega)\right)$ norm obtained with $k=3, \nu=10^{-3}$ and $\mathcal{T}_{h}^{4}$.

\begin{tabular}{|c|c|c|c|c|c|c|}
\hline dof & $h$ & $\Delta t_{0}$ & $\Delta t_{0} / 2$ & $\Delta t_{0} / 4$ & $\Delta t_{0} / 8$ & $\Delta t_{0} / 16$ \\
\hline 211 & $h_{0}$ & $9.546949 \mathrm{e}-4$ & $9.395878 \mathrm{e}-4$ & $9.320480 \mathrm{e}-4$ & $9.282763 \mathrm{e}-4$ & $9.263893 \mathrm{e}-4$ \\
\hline 899 & $h_{0} / 2$ & $2.760235 \mathrm{e}-4$ & $2.701636 \mathrm{e}-4$ & $2.678057 \mathrm{e}-4$ & $2.667518 \mathrm{e}-4$ & $2.662540 \mathrm{e}-4$ \\
\hline 3715 & $h_{0} / 4$ & $8.270615 \mathrm{e}-5$ & $7.376976 \mathrm{e}-5$ & \begin{tabular}{|l|}
$7.135559 \mathrm{e}-5$ \\
\end{tabular} & $7.064923 \mathrm{e}-5$ & $7.041604 \mathrm{e}-5$ \\
\hline 15107 & $h_{0} / 8$ & $4.423838 \mathrm{e}-5$ & $2.603643 \mathrm{e}-5$ & $1.969686 \mathrm{e}-5$ & \begin{tabular}{|l|}
$1.785950 \mathrm{e}-5$ \\
\end{tabular} & $1.736430 \mathrm{e}-5$ \\
\hline 60931 & $h_{0} / 16$ & $4.077910 \mathrm{e}-5$ & $1.976831 \mathrm{e}-5$ & $1.021248 \mathrm{e}-5$ & $6.047226 \mathrm{e}-6$ & $4.554491 \mathrm{e}-6$ \\
\hline
\end{tabular}

We consider the time interval $[0,1]$, for the viscosity we consider the values $\nu=1$ and $\nu=10^{-3}$ and we start the process with $h_{0}=1 / 8$ and $\Delta t_{0}=1 / 16$.

We report in Tables 1 and 2 the errors $\mathrm{E}_{2}(\psi)$ for different refinement levels and time steps and using the family of meshes $\mathcal{T}_{h}^{2}$ for $k=2$ and the family $\mathcal{T}_{h}^{4}$ for $k=3$. Moreover, the maximum number of iterations that are required for the Newton method in this example is 4 for all the meshes and for $k=2,3$. It can be seen along the diagonal of Table 1, that the error $\mathrm{E}_{2}(\psi)$ reduces linearly with respect to $h$ and along the diagonal of Table 2 can be observed that the error $\mathrm{E}_{2}(\psi)$ reduces quadratically with respect to $h$, which are the expected order of convergence for $k=2$ and $k=3$, respectively. In addition, we have highlighted the errors which are dominated by space in Tables 1 and 2 for small values of time-step $\Delta t$. We also observed that for big values of $h$, the error $\mathrm{E}_{2}(\psi)$ is almost constant with respect to $\Delta t$. Further, to examine the rate of convergence for the space variable, we have included convergence graph using all family of meshes in Figure 2, for $k=2$ and $k=3$.

\subsection{Test 2. Non-homogeneous Dirichlet boundary conditions and initial conditions}

In this numerical test, we solve the Navier-Stokes problem $(2.1)$ on the square domain $\Omega:=(0,1)^{2}$. We take the load term $\mathbf{f}$, non-homogeneous Dirichlet boundary and initial conditions in such a way that the analytical solution is given by:

$$
\begin{aligned}
& \boldsymbol{u}(x, y, t)=0.1 \pi\left(\begin{array}{r}
-\exp (0.1 t)(1+\cos (\pi x)) \sin (\pi y) \\
\exp (0.1 t)(1+\cos (\pi y)) \sin (\pi x)
\end{array}\right), \quad p(x, y, t)=\exp (-t)\left(x^{2}+y^{2}-\frac{2}{3}\right) \\
& \psi(x, y, t)=0.1 \exp (0.1 t)(1+\cos (\pi x))(1+\cos (\pi y)) .
\end{aligned}
$$

In this test, we consider the time interval to $[0,1]$, the viscosity $\nu=10^{-3}$ and we start with $h_{0}=1 / 4$ and $\Delta t_{0}=1 / 16$. 

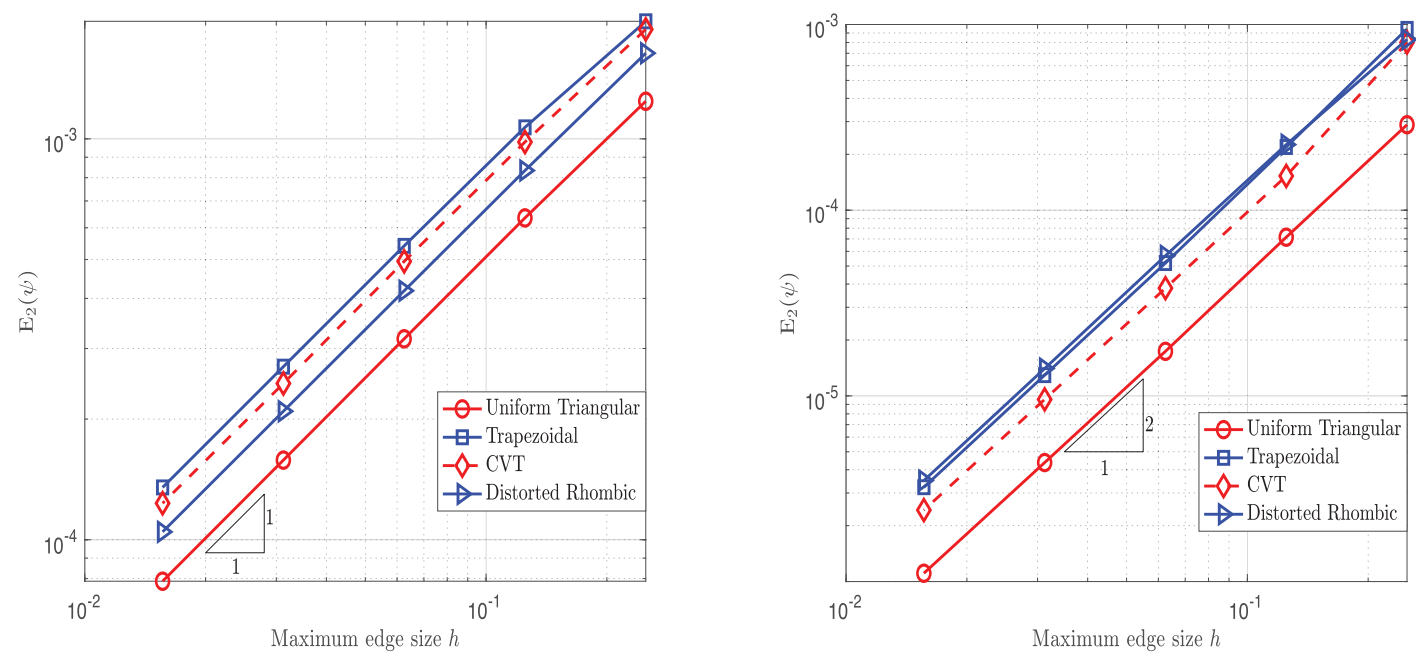

FiguRE 2. Test 1: Rate of convergence for space variable for different meshes with meshsize $h=1 / 4, \ldots, 1 / 64, \nu=1$ and for small values of time-step $\Delta t$. For $k=2$ and $\Delta t=0.05$ (left panel) and $k=3$ and $\Delta t=0.0025$ (right panel).

TABLE 3. Test 2. Errors for the stream-function $\psi_{h}$ in the discrete $L^{2}\left(0, T ; H^{2}(\Omega)\right)$ norm obtained with $\mathcal{T}_{h}^{4}$ and $\nu=10^{-3}$.

\begin{tabular}{|c|c|c|c|c|c|c|}
\hline dof & $h$ & $\Delta t_{0}$ & $\Delta t_{0} / 2$ & $\Delta t_{0} / 4$ & $\Delta t_{0} / 8$ & $\Delta t_{0} / 16$ \\
\hline 123 & $h_{0}$ & \begin{tabular}{|l|l}
$3.312118 \mathrm{e}-1$ \\
\end{tabular} & $3.307043 \mathrm{e}-1$ & $3.304508 \mathrm{e}-1$ & $3.303242 \mathrm{e}-1$ & $3.302609 \mathrm{e}-1$ \\
\hline 531 & $h_{0} / 2$ & $1.674332 \mathrm{e}-1$ & $1.672076 \mathrm{e}-1$ & $1.670951 \mathrm{e}-1$ & $1.670389 \mathrm{e}-1$ & $\begin{array}{l}1.670108 \mathrm{e}-1 \\
\end{array}$ \\
\hline 2211 & $h_{0} / 4$ & $8.358238 \mathrm{e}-2$ & $8.345864 \mathrm{e}-2$ & $8.339822 \mathrm{e}-2$ & $8.336843 \mathrm{e}-2$ & $8.335366 \mathrm{e}-2$ \\
\hline 9027 & $h_{0} / 8$ & $4.229862 \mathrm{e}-2$ & $4.222367 \mathrm{e}-2$ & $4.218726 \mathrm{e}-2$ & \begin{tabular}{|l|}
$4.216934 \mathrm{e}-2$ \\
\end{tabular} & $4.216047 \mathrm{e}-2$ \\
\hline 36483 & $h_{0} / 16$ & $2.163267 \mathrm{e}-2$ & $2.160239 \mathrm{e}-2$ & $2.158950 \mathrm{e}-2$ & $2.158367 \mathrm{e}-2$ & $2.158092 \mathrm{e}-2$ \\
\hline
\end{tabular}

We report in Table 3 the errors $\mathrm{E}_{2}(\psi)$ for the family of meshes $\mathcal{T}_{h}^{4}$ and different refinement levels and time steps. In this example, the maximum number of iterations that are required for the Newton method is 5 . Once again, it can be seen along the diagonal of Table 3 that the error $\mathrm{E}_{2}(\psi)$ reduces linearly with respect to $h$, which is the expected order of convergence for $k=2$. In addition, we have highlighted the errors which are dominated by space in Table 3 for small values of time-step $\Delta t$.

\subsection{Test 3. Example with dominating time error}

In the present numerical test, we study the order of convergence of the virtual scheme in time. We solve the Navier-Stokes problem $(2.1)$ on the square domain $\Omega:=(0,1)^{2}$. We take the load term $\mathbf{f}$, boundary and initial conditions in such a way that the analytical solution is given by:

$$
\begin{aligned}
& \boldsymbol{u}(x, y, t)=\left(\begin{array}{r}
\sin (10 \pi t) x^{2}(1-x)^{2}\left(2 y-6 y^{2}+4 y^{3}\right) \\
-\sin (10 \pi t) y^{2}(1-y)^{2}\left(2 x-6 x^{2}+4 x^{3}\right)
\end{array}\right), \\
& p(x, y, t)=-\left(x^{3}+y^{3}-\frac{1}{2}\right)\left(\frac{3}{2}+\frac{1}{2} \sin (10 \pi t)\right), \quad \psi(x, y, t)=\sin (10 \pi t) x^{2}(1-x)^{2} y^{2}(1-y)^{2} .
\end{aligned}
$$

We consider the time interval $[0,1]$, the viscosity $\nu=1$ and we start the process with $h_{0}=1 / 8$ and $\Delta t_{0}=1 / 8$. 
TABle 4. Test 3. Errors and experimental rates for the stream-function $\psi_{h}$ in norm $L^{2}\left(0, T ; H^{2}(\Omega)\right)$ obtained with the meshes $\mathcal{T}_{h}^{1}, k=2$ and $\nu=1$.

\begin{tabular}{|c|c|c|c|c|c|c|}
\hline dof & $h$ & $\Delta t_{0}$ & $\Delta t_{0} / 2$ & $\Delta t_{0} / 4$ & $\Delta t_{0} / 8$ & $\Delta t_{0} / 16$ \\
\hline 147 & $h_{0}$ & $2.556643 \mathrm{e}-2$ & $1.851436 \mathrm{e}-2$ & $1.439994 \mathrm{e}-2$ & $1.256422 \mathrm{e}-2$ & $1.186603 \mathrm{e}-2$ \\
\hline 675 & $h_{0} / 2$ & $2.437394 \mathrm{e}-2$ & $1.579445 \mathrm{e}-2$ & $1.025426 \mathrm{e}-2$ & $7.432721 \mathrm{e}-3$ & $6.296528 \mathrm{e}-3$ \\
\hline 2883 & $h_{0} / 4$ & $2.406341 \mathrm{e}-2$ & $1.502749 \mathrm{e}-2$ & \begin{tabular}{|l|}
$8.911963 \mathrm{e}-3$ \\
\end{tabular} & $5.428423 \mathrm{e}-3$ & $3.772418 \mathrm{e}-3$ \\
\hline 11907 & $h_{0} / 8$ & $2.398373 \mathrm{e}-2$ & $1.482783 \mathrm{e}-2$ & $8.541759 \mathrm{e}-3$ & $4.795144 \mathrm{e}-3$ & $2.800171 \mathrm{e}-3$ \\
\hline 48387 & $h_{0} / 16$ & $2.396363 \mathrm{e}-2$ & $1.477733 \mathrm{e}-2$ & $8.446522 \mathrm{e}-3$ & $4.623010 \mathrm{e}-3$ & $2.498059 \mathrm{e}-3$ \\
\hline
\end{tabular}

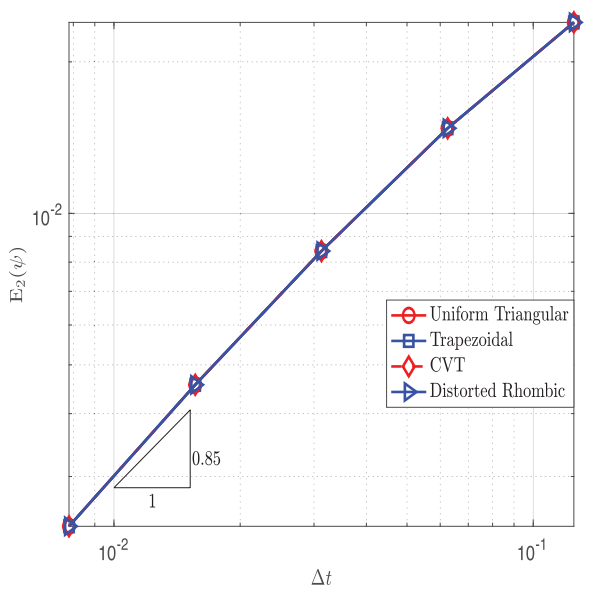

FiguRE 3. Rate of convergence for time variable for different meshes with time-steps $\Delta t=$ $1 / 8, \ldots, 1 / 128$ and meshsize $h=1 / 64, \nu=1$ and $k=3$.

We report in Table 4 the errors $\mathrm{E}_{2}(\psi)$ obtained for $k=2$ and using the family of meshes $\mathcal{T}_{h}^{1}$ with different refinement levels and time steps. In this experiment, differently to the first and second tests, we can observe that, for small values of $h$, the error $\mathrm{E}_{2}(\psi)$ reduces linearly with respect to $\Delta t$ (see the last row of Tab. 4), which is the expected order of convergence in time according to Theorem 5.6. We also observe that for big values of $\Delta t$ the error $\mathrm{E}_{2}(\psi)$ is almost constant with respect to $h$. In this numerical test, the maximum number of iterations that are required for the Newton method is 3 .

Further, in Figure 3, we have posted a convergence graph where errors $\mathrm{E}_{2}(\psi)$ are dominated by time and virtual element space of order $k=3$ is chosen. We have used all family of meshes. We deduce that the rate of convergence is closer to 1 (expected order of convergence for time variable) for very small values of $h$.

\subsection{Test 4. Chorin problem}

In this example, we consider the well-known Chorin problem for incompressible Navier-Stokes equations [22]. For this test the load term is $\mathbf{f}=\mathbf{0}$, and the initial and boundary conditions correspond to the analytical solution:

$$
\begin{aligned}
& \boldsymbol{u}(x, y, t)=\left(\begin{array}{r}
-\cos (2 \pi x) \sin (2 \pi y) e^{-8 \pi^{2} \nu t} \\
\sin (2 \pi x) \cos (2 \pi y) e^{-8 \pi^{2} \nu t}
\end{array}\right), \quad p(x, y, t)=-\frac{1}{4}(\cos (4 \pi x)+\cos (4 \pi y)) e^{-8 \pi^{2} \nu t}, \\
& \psi(x, y, t)=\frac{1}{2 \pi} \cos (2 \pi x) \cos (2 \pi y) e^{-8 \pi^{2} \nu t} .
\end{aligned}
$$


TABLE 5. Test 4. Errors and experimental rates for the stream-function $\psi_{h}$, using the meshes $\mathcal{T}_{h}^{3}$, and with different values of $\nu$.

\begin{tabular}{lllllll}
\hline \hline$\nu$ & dof & $h$ & $\mathrm{E}_{1}(\psi)$ & $\mathrm{r}_{1}(\psi)$ & $\mathrm{E}_{2}(\psi)$ & $\mathrm{r}_{2}(\psi)$ \\
\hline \multirow{2}{*}{$10^{-3}$} & 54 & $1 / 4$ & $2.589181 \mathrm{e}-2$ & - & $3.689706 \mathrm{e}-1$ & - \\
& 1371 & $1 / 8$ & $6.856293 \mathrm{e}-3$ & 1.916 & $1.979657 \mathrm{e}-1$ & 0.898 \\
& 5796 & $1 / 32$ & $4.796994 \mathrm{e}-3$ & 1.931 & $1.012519 \mathrm{e}-1$ & 0.967 \\
& 23874 & $1 / 64$ & $1.078390 \mathrm{e}-4$ & 2.006 & $2.519974 \mathrm{e}-2$ & 1.005 \\
& & & & & & \\
10 & 54 & $1 / 4$ & $2.590922 \mathrm{e}-2$ & - & $3.691334 \mathrm{e}-1$ & - \\
& 294 & $1 / 8$ & $6.858939 \mathrm{e}-3$ & 1.917 & $1.980514 \mathrm{e}-1$ & 0.898 \\
& 1371 & $1 / 16$ & $1.796887 \mathrm{e}-3$ & 1.932 & $1.012955 \mathrm{e}-1$ & 0.967 \\
& 5796 & $1 / 32$ & $4.328584 \mathrm{e}-4$ & 2.053 & $5.061318 \mathrm{e}-2$ & 1.000 \\
& 23874 & $1 / 64$ & $1.074008 \mathrm{e}-4$ & 2.010 & $2.520898 \mathrm{e}-2$ & 1.005 \\
\hline
\end{tabular}

It has been observed that some finite element methods for a velocity-pressure formulation, the $L^{2}$ error of the velocity converge suboptimally or even lock for small values of the viscosity (see [32], Sect. 4.2). It can be seen that the $L^{2}$ error of the velocity is related with the $H^{1}$ error for the stream-function. Thus, in order to assess the performance of the virtual scheme for this numerical example, we introduce the following discrete quantity:

$$
\mathrm{E}_{1}(\psi):=\operatorname{error}\left(\psi, L^{2}, H^{1}\right)=\left(\Delta t \sum_{n=1}^{N}\left|\psi\left(t_{n}\right)-\Pi_{K}^{k, \Delta} \psi_{h}^{n}\right|_{1, h}^{2}\right)^{1 / 2} .
$$

We observe that an additional order of convergence is expected in this discrete error. To show this fact, we will compute experimental rates of convergence for each individual error as follows:

$$
\mathrm{r}_{i}(\psi):=\frac{\log \left(\mathrm{E}_{i}(\psi) / \mathrm{E}_{i}^{\prime}(\psi)\right)}{\log \left(h / h^{\prime}\right)}, \quad i=1,2,
$$

where $h, h^{\prime}$ denote two consecutive mesh sizes with their respective errors $\mathrm{E}_{i}$ and $\mathrm{E}_{i}^{\prime}$.

We report in Table 5 the discrete errors $\mathrm{E}_{1}(\psi)$ and $\mathrm{E}_{2}(\psi)$, for the family of meshes $\mathcal{T}_{h}^{3}$. The results were obtained by considering the final time $T=0.01$ and time stepping $\Delta t=0.001$. For the viscosity $\nu$, we take two values: $\nu=10^{-3}$ and $\nu=10^{-6}$. The maximum number of iterations that are required for the Newton method in this example is 4 for $\nu=10^{-3}$ and 5 for $\nu=10^{-6}$.

It can be clearly observed from Table 5 a linear order of convergence in the norm $\mathrm{E}_{2}(\psi)$ and a quadratic order in the norm $\mathrm{E}_{1}(\psi)$ (which has not been proved). Thus, we conclude that our virtual scheme does not suffer from a suboptimal order of convergence or locking phenomenon.

Exact and approximate solutions (including a postprocessed velocity field) are illustrated in Figure 4.

\section{Conclusion}

In this article, we have proposed a $C^{1}$ VEM to discretize the time-dependent Navier-Stokes problem in the stream-function form. Exploiting enhanced virtual element spaces, we have approximated the spatial variables and we have discretized the time variable using the backward Euler scheme. We have derived a priori error estimations for semi-discrete and fully-discrete schemes and the theoretical results are verified by four numerical experiments. Moreover, the fourth numerical experiment allows us to conclude that our virtual scheme does not suffer from a suboptimal order of convergence when the diffusion coefficient $\nu$ is small, in contrast to some finite element methods in velocity-pressure formulation, where a suboptimal convergence is observed in $L^{2}$-norm of the velocity for the Chorin problem with small values of $\nu$ (see [32]). 

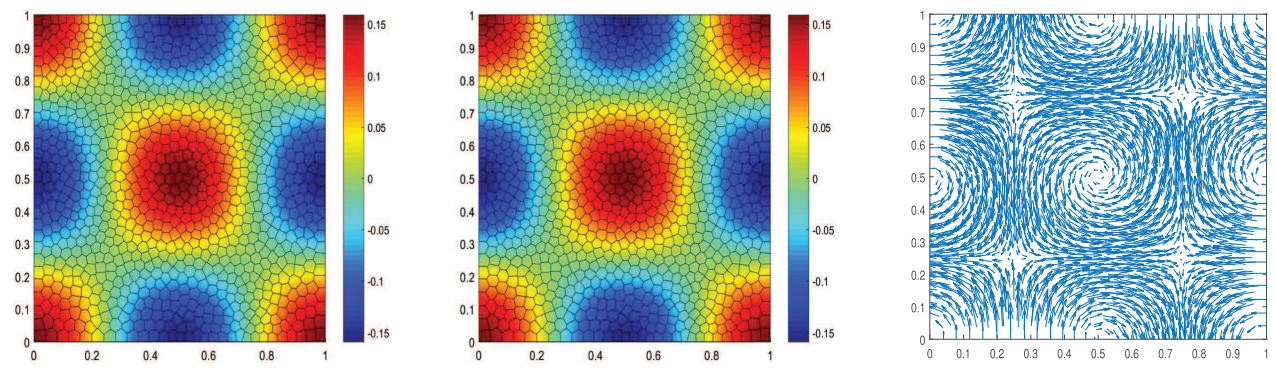

Figure 4 . Test 4 . Exact and approximate solutions $\psi, \psi_{h}$ and the postprocessed velocity field $\boldsymbol{u}_{h}:=\operatorname{curl} \psi_{h}$, using $\Delta t=0.001$ for $T=0.01$, using the mesh $\mathcal{T}_{h}^{3}$ with $h=1 / 32$ and $\nu=10^{-6}$.

Acknowledgements. The first author was partially supported by the National Agency for Research and Development, ANID-Chile through FONDECYT Postdoctorado project 3200242 (Chile). The second author was partially supported by the National Agency for Research and Development, ANID-Chile through FONDECYT project 1180913 and by project AFB170001 of the PIA Program: Concurso Apoyo a Centros Científicos y Tecnológicos de Excelencia con Financiamiento Basal. The fourth author was supported by the National Agency for Research and Development, ANID-Chile, Scholarship Program, Doctorado Becas Chile 2020, 21201910.

\section{REFERENCES}

[1] D. Adak and S. Natarajan, On the $H^{1}$ conforming virtual element method for time dependent Stokes equation. Math. Comput. Sci. 15 (2021) 135-154.

[2] R.A. Adams and J.J.F. Fournier, Sobolev Spaces, 2nd edition. In: Vol. 140 of Pure and Applied Mathematics. Academic Press, New York (2003).

[3] B. Ahmad, A. Alsaedi, F. Brezzi, L.D. Marini and A. Russo, Equivalent projectors for virtual element methods. Comput. Math. App. 66 (2013) 376-391.

[4] P.F. Antonietti, L. Beirão da Veiga, D. Mora and M. Verani, A stream virtual element formulation of the Stokes problem on polygonal meshes. SIAM J. Numer. Anal. 52 (2014) 386-404.

[5] P.F. Antonietti, L. Beirão da Veiga, S. Scacchi and M. Verani, A $C^{1}$ virtual element method for the Cahn-Hilliard equation with polygonal meshes. SIAM J. Numer. Anal. 54 (2016) 36-56.

[6] L. Beirão da Veiga, F. Brezzi, A. Cangiani, G. Manzini, L.D. Marini and A. Russo, Basic principles of virtual element methods. Math. Models Methods Appl. Sci. 23 (2013) 199-214.

[7] L. Beirão da Veiga, F. Brezzi, L.D. Marini and A. Russo, The hitchhiker's guide to the virtual element method. Math. Models Methods Appl. Sci. 24 (2014) 1541-1573.

[8] L. Beirão da Veiga, C. Lovadina and G. Vacca, Divergence free virtual elements for the Stokes problem on polygonal meshes. ESAIM: M2AN 51 (2017) 509-535.

[9] L. Beirão da Veiga, C. Lovadina and G. Vacca, Virtual elements for the Navier-Stokes problem on polygonal meshes. SIAM J. Numer. Anal. 56 (2018) 1210-1242.

[10] L. Beirão da Veiga, D. Mora and G. Rivera, Virtual elements for a shear-deflection formulation of Reissner-Mindlin plates. Math. Comput. 88 (2019) 149-178.

[11] L. Beirão da Veiga, D. Mora and G. Vacca, The Stokes complex for virtual elements with application to Navier-Stokes flows. J. Sci. Comput. 81 (2019) 990-1018.

[12] L. Beirão da Veiga, F. Dassi and A. Russo, A $C^{1}$ virtual element method on polyhedral meshes. Comput. Math. App. 79 (2020) 1936-1955.

[13] L. Beirão da Veiga, F. Dassi and G. Vacca, The Stokes complex for virtual elements in three dimensions. Math. Models Methods Appl. Sci. 30 (2020) 477-512.

[14] G. Ben-Yu, H. Li-Ping and M. De-Kang, On the two-dimensional Navier-Stokes equations in stream function form. J. Math. Anal. App. 205 (1997) 1-31.

[15] S.C. Brenner and R.L. Scott, The Mathematical Theory of Finite Element Methods. Springer, New York (2008).

[16] F. Brezzi and L.D. Marini, Virtual elements for plate bending problems. Comput. Methods Appl. Mech. Eng. 253 (2013) 455-462.

[17] E. Cáceres and G.N. Gatica, A mixed virtual element method for the pseudostress-velocity formulation of the Stokes problem. IMA J. Numer. Anal. 37 (2017) 296-331. 
[18] A. Cangiani, V. Gyrya and G. Manzini, The nonconforming virtual element method for the Stokes equations. SIAM J. Numer. Anal. 54 (2016) 3411-3435.

[19] A. Cangiani, G. Manzini and O.J. Sutton, Conforming and nonconforming virtual element methods for elliptic problems. IMA J. Numer. Anal. 37 (2017) 1317-1354.

[20] M. Cayco and R.A. Nicolaides, Finite element technique for optimal pressure recovery from stream function formulation of viscous flows. Math. Comput. 46 (1986) 371-377.

[21] C. Chinosi and L.D. Marini, Virtual element method for fourth order problems: $L^{2}$-estimates. Comput. Math. App. 72 (2016) 1959-1967.

[22] A.J. Chorin, Numerical solution for the Navier-Stokes equations. Math. Comput. 22 (1968) 745-762.

[23] P.G. Ciarlet, The Finite Element Method for Elliptic Problems. SIAM (2002).

[24] F. Dassi and G. Vacca, Bricks for the mixed high-order virtual element method: projectors and differential operators. Appl. Numer. Math. 155 (2020) 140-159.

[25] E. Foster, T. Iliescu and Z. Wang, A finite element discretization of the stream function formulation of the stationary quasigeostrophic equations of the ocean. Comput. Methods Appl. Mech. Eng. 261/262 (2013) 105-117.

[26] G.N. Gatica, M. Munar and F. Sequeira, A mixed virtual element method for the Navier-Stokes equations. Math. Models Methods Appl. Sci. 28 (2018) 2719-2762.

[27] V. Girault and P.A. Raviart, Finite Element Approximation of the Navier-Stokes Equations, 749. Springer-Verlag, Berlin-New York (1979).

[28] V. Girault and P.A. Raviart, Finite Element Methods for Navier-Stokes Equations. Springer-Verlag, Berlin (1986).

[29] P. Grisvard, Elliptic Problems in Non-Smooth Domains. Pitman, Boston (1985).

[30] D. Irisarri and G. Hauke, Stabilized virtual element methods for the unsteady incompressible Navier-Stokes equations. Calcolo 56 (2019) 1-21.

[31] M.J. Lai, C. Liu and P. Wenston, Bivariate spline method for numerical solution of time evolution Navier-Stokes equations over polygons in stream function formulation. Numer. Methods Part. Diff. Equ. 19 (2003) 776-827.

[32] A. Linke and L.G. Rebholz, Pressure-induced locking in mixed methods for time-dependent (Navier-)Stokes equations. J. Comput. Phys. 388 (2019) 350-356.

[33] X. Liu and Z. Chen, The nonconforming virtual element method for the Navier-Stokes equations. Adv. Comput. Math. 45 (2019) 51-74

[34] X. Liu, R. Li and Z. Chen, A virtual element method for the coupled Stokes-Darcy problem with the Beaver-Joseph-Saffman interface condition. Calcolo 56 (2019) 1-28.

[35] D. Mora and I. Velásquez, Virtual element for the buckling problem of Kirchhoff-Love plates. Comput. Methods Appl. Mech. Eng. 360 (2020) 112687.

[36] D. Mora, G. Rivera and I. Velásquez, A virtual element method for the vibration problem of Kirchhoff plates. ESAIM: M2AN 52 (2018) 1437-1456.

[37] A. Quarteroni and A. Valli, Numerical Aproximation of the Partial Differential Equation. Springer-Verlag Berlin Heidelberg (1994).

[38] R. Temam, Navier-Stokes Equations. North-Holland, Amsterdam (1977).

[39] G. Vacca, An $H^{1}$-conforming virtual element for Darcy and Brinkman equations. Math. Models Methods Appl. Sci. 28 (2018) 159-194.

[40] G. Vacca and L. Beirão da Veiga, Virtual element methods for parabolic problems on polygonal meshes. Numer. Methods Part. Diff. Equ. 31 (2015) 2110-2134.

[41] J. Zhao, B. Zhang, S. Mao and S. Chen, The divergence-free nonconforming virtual element for the Stokes problem. SIAM J. Numer. Anal. 57 (2019) 2730-2759.

\section{Subscribe to Open (S20) A fair and sustainable open access model}

This journal is currently published in open access under a Subscribe-to-Open model (S2O). S2O is a transformative model that aims to move subscription journals to open access. Open access is the free, immediate, online availability of research articles combined with the rights to use these articles fully in the digital environment. We are thankful to our subscribers and sponsors for making it possible to publish this journal in open access, free of charge for authors.

\section{Please help to maintain this journal in open access!}

Check that your library subscribes to the journal, or make a personal donation to the S2O programme, by contacting subscribers@edpsciences.org

More information, including a list of sponsors and a financial transparency report, available at: https://www. edpsciences.org/en/maths-s2o-programme 